\title{
Probing Single-Particle Electrocatalytic Activity at Facet-Controlled Gold Nanocrystals
}

Myunghoon Choi, $\neq$ Natasha P. Siepser,, Soojin Jeong,,+ Yi Wang, Gargi Jagdale, Xingchen Ye*, and Lane A. Baker*

Department of Chemistry, Indiana University, 800 E. Kirkwood Avenue, Bloomington, Indiana 47405

KEYWORDS : scanning electrochemical cell microscopy, SECCM, nanocubes, nano-octahedra, single-particle catalysis, facet dependent catalytic activity. 


\section{Supporting Information}

Section S1. Scale Drawing of SECCM Droplet Cell at Single Gold Nanocubes and Nano-

Octahedra

Section S2. Synthesis of Gold Nanocubes and Nano-Octahedra

Section S3. Preparation of Working Electrodes

Section S4. Scanning Electrochemical Cell Microscopy (SECCM) Operation and Instrumentation

Section S5. SECCM Cyclic Voltammetric Mapping for Reduction Reactions (HER and ORR)

Section S6. Finite Element Method (FEM) Simulations and Experimental Result Comparison of SECCM CV Measured at Individual Particles

Supplementary References 


\section{Section S1. Scale Drawing of SECCM Cell at Single Gold Nanocubes}

\section{and Nano-Octahedra}
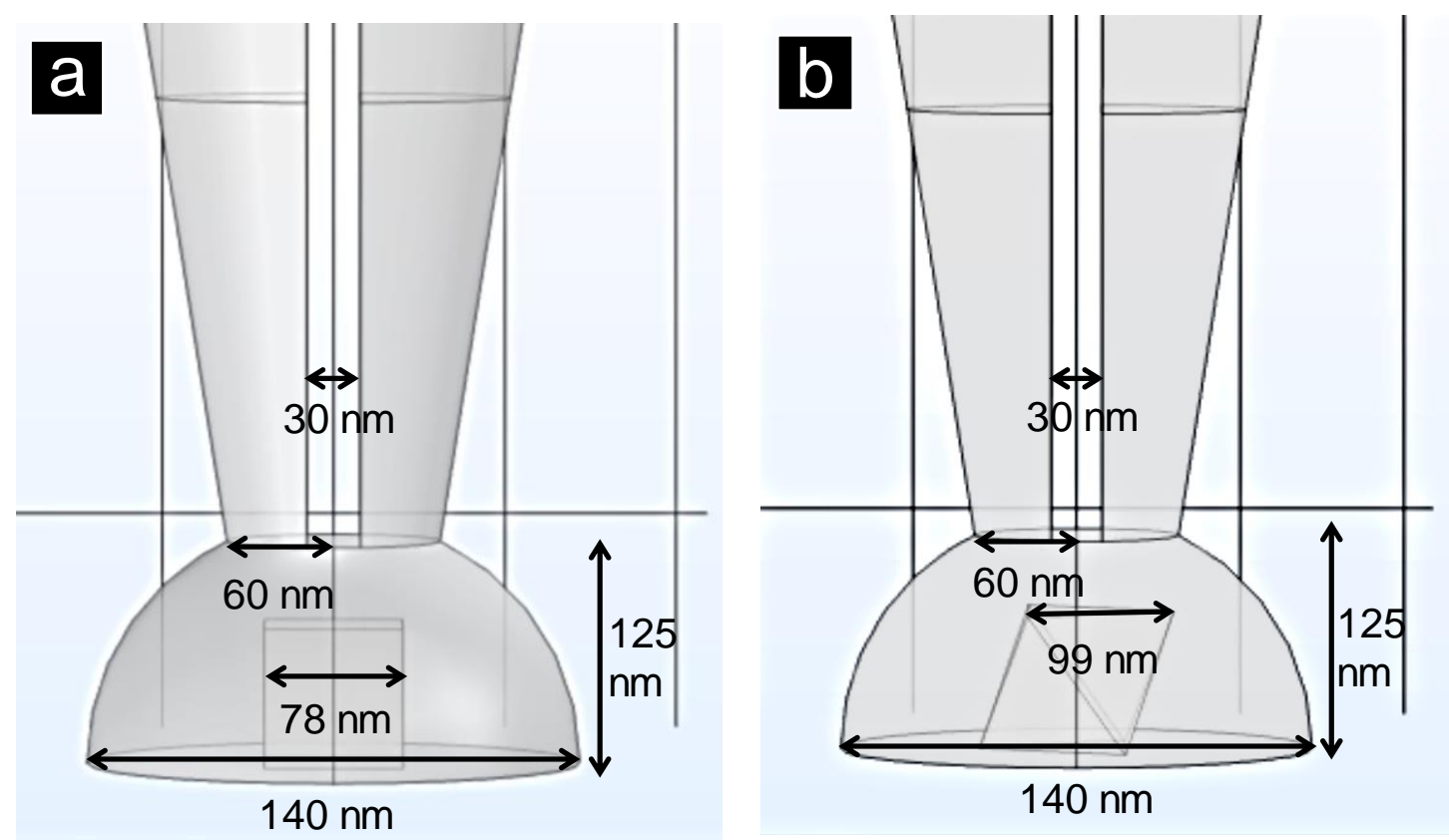

Figure S1. Scale drawing of SECCM droplet cell dimensions for finite element modeling when the $\mathrm{Au} \mathrm{NC}$ (a) and $\mathrm{Au} \mathrm{OD}$ (b) were centered in the drop. The inner and outer diameter of the pipette was ca. $120 \mathrm{~nm}$ and $200 \mathrm{~nm}$, respectively. 


\section{Section S2. Synthesis of Gold Nanocubes and Nano-Octahedra}

\subsection{Reagents}

Hydrogen tetrachloroaurate trihydrate $\left(\mathrm{HAuCl}_{4} \cdot 3 \mathrm{H}_{2} \mathrm{O}, \geq 99.9 \%\right.$ trace metals basis), sodium borohydride $\left(\mathrm{NaBH}_{4}, 99 \%\right)$, silver nitrate $\left(\mathrm{AgNO}_{3}, \geq 99.0 \%\right)$, L-ascorbic acid $(\geq 99.5 \%)$, hydrochloric acid $(\mathrm{HCl}, 37 \%)$ and nitric acid $\left(\mathrm{HNO}_{3}, 70 \%\right)$ were purchased from Sigma Aldrich. Hexadecyltrimethylammonium bromide $(\mathrm{CTAB},>98.0 \%)$ and hexadecylpyridinium chloride monohydrate $(\mathrm{CPC},>98.0 \%)$ were purchased from TCI America. Potassium bromide $(\mathrm{KBr}$, $99.999 \%$ ) was purchased from Acros Organics. All chemicals were used as received without further purification. Ultrapure water (resistivity $=18.2 \mathrm{M} \Omega \cdot \mathrm{cm}$ at $25^{\circ} \mathrm{C}$ ) obtained from a Barnstead ${ }^{\circledR}$ GenPure $^{\circledR}$ (Thermo Scientific) or Milli-Q (MilliporeSigma) water purification system were used in all experiments. All glassware was cleaned with aqua regia (a mixture of $\mathrm{HCl}$ and $\mathrm{HNO}_{3}$ in 3:1 volume ratio), rinsed thoroughly with water and dried before use.

\subsection{Synthesis of Au Nanorods}

Au nanorods (NRs) were synthesized by using a seed-mediated growth method. ${ }^{1}$ First, 125 $\mu \mathrm{L}$ of $10 \mathrm{mM} \mathrm{HAuCl}_{4}$ solution was added to $5 \mathrm{~mL}$ of $100 \mathrm{mM} \mathrm{CTAB}$ solution, to which freshly prepared $300 \mu \mathrm{L}$ of $\mathrm{NaBH}_{4}$ solution $(10 \mathrm{mM})$ was rapidly injected under vigorous stirring. This seed solution was aged at room temperature for 1 hour. The growth solution was prepared by adding separately prepared solutions of $10 \mathrm{mM} \mathrm{HAuCl}_{4}(10 \mathrm{~mL}), 10 \mathrm{mM} \mathrm{AgNO}_{3}(1.8 \mathrm{~mL})$ and $100 \mathrm{mM}$ L-ascorbic acid $(1.2 \mathrm{~mL})$ into $200 \mathrm{~mL}$ of CTAB solution $(100 \mathrm{mM})$. Afterwards, $240 \mu \mathrm{L}$ of aged seed solution was injected into the colorless growth solution. The reaction mixture was stirred for another $30 \mathrm{~s}$ and was left undisturbed at $30{ }^{\circ} \mathrm{C}$ for 5 hours. The product was isolated via 
centrifugation at $8000 \mathrm{rpm}$ for $10 \mathrm{~min}$. After two rounds of dissolution in $50 \mathrm{mM} \mathrm{CTAB}$ followed by centrifugation, Au NRs were dispersed in an appropriate volume of $50 \mathrm{mM}$ CTAB solution so that the optical density (O.D.) reached 2.0 at the longitudinal plasmon peak wavelength.

\subsection{Synthesis of Spherical Au Seeds}

$\mathrm{Au}$ nanospheres were synthesized by using an iterative oxidative dissolution and reductive growth method developed by the Mirkin group. ${ }^{2}$ First, etching of Au NRs was initiated by adding $10 \mathrm{mM} \mathrm{HAuCl}_{4}$ into as-synthesized NR solution (O.D. $\left.=2\right)$ to obtain a final concentration of 75 $\mu \mathrm{M}$ for $\mathrm{Au}^{3+}$. The reaction was allowed to proceed for 3 hours at $40{ }^{\circ} \mathrm{C}$ under stirring. The products were retrieved by centrifugation at $8000 \mathrm{rpm}$ for $10 \mathrm{~min}$ followed by dissolution with $100 \mathrm{mM}$ CPC to attain O.D. $=1.0$. Afterwards, Au concave rhombic dodecahedra (CRDs) were synthesized by using these etched NRs as seeds. In a typical reaction, $3 \mathrm{~mL}$ of etched NR solution in CPC was added into a mixture prepared from $10 \mathrm{~mL}$ of $10 \mathrm{mM} \mathrm{CPC}, 175 \mu \mathrm{L}$ of $10 \mathrm{mM} \mathrm{HAuCl}_{4}$ and 2.25 $\mathrm{mL}$ of $100 \mathrm{mM} \mathrm{L}$-ascorbic acid. After stirring at $500 \mathrm{rpm}$ for $15 \mathrm{~min}$, the reaction was halted by centrifugation at $8000 \mathrm{rpm}$ for $10 \mathrm{~min}$. The CRDs were dispersed into a certain volume of $50 \mathrm{mM}$ CTAB solution to reach O.D. $=1$. Subsequent etching of these CRDs were induced by adding 10 $\mathrm{mM} \mathrm{HAuCl}_{4}$ until a final concentration of $60 \mu \mathrm{M}$ is reached for $\mathrm{Au}^{3+}$. The reaction solution was kept at $40{ }^{\circ} \mathrm{C}$ for $4-6$ hours under stirring and the nanospheres were isolated by centrifugation at $8000 \mathrm{rpm}$ for $10 \mathrm{~min}$. The size of final spherical seed particles were controlled by monitoring the extent of etching via UV-VIS extinction spectroscopy at 30-min. intervals. For example, the reaction was stopped after 4.5 hours once the plasmonic absorption peak reached $524.0 \mathrm{~nm}$. The resultant nanospheres with an average diameter of ca. $19 \mathrm{~nm}$ were used as seeds for the preparation of Au nanocubes used in this work. On the other hand, the $37.0 \mathrm{~nm}$ seed particles used for the 
synthesis of $\mathrm{Au}$ octahedra were obtained after 6 hours of etching when the plasmonic absorption peak reached $526.5 \mathrm{~nm}$.

\subsection{Synthesis of Au Nanocubes}

Au nanocubes were synthesized by using a previously reported seeded-growth method with

slight modifications. ${ }^{3}$ Firstly, 19-nm seed particles were dissolved with $100 \mathrm{mM} \mathrm{CPC}$ solution to attain O.D. $=1.0$. The growth solution was prepared by mixing $25 \mathrm{~mL}$ of $100 \mathrm{mM} \mathrm{CPC}, 2.5 \mathrm{~mL}$ of $100 \mathrm{mM} \mathrm{KBr}, 1.5 \mathrm{~mL}$ of $10 \mathrm{mM} \mathrm{HAuCl}_{4}$ and $2.25 \mathrm{~mL}$ of $100 \mathrm{mM} \mathrm{L}$-ascorbic acid solutions. Afterwards, $750 \mu \mathrm{L}$ of the above seed solution was introduced and the reaction mixture was left undisturbed for 1 hour. Au nanocubes were purified by two rounds of centrifugation at $3000 \mathrm{rpm}$ for $3 \mathrm{~min}$ and dissolution in $25 \mathrm{~mL}$ of $50 \mathrm{mM}$ CTAB solution. Au nanocubes were finally dispersed in water at the concentration of O.D. $=2.0$.

\subsection{Synthesis of Au Nano-Octahedra}

Au octahedra were synthesized according to a seed-growth method reported previously. ${ }^{2}$ Firstly, 19-nm Au seed particles were dissolved with $100 \mathrm{mM} \mathrm{CPC}$ solution to obtain O.D. = 1.0. The growth solution was prepared by mixing $25 \mathrm{~mL}$ of $100 \mathrm{mM} \mathrm{CPC}, 500 \mu \mathrm{L}$ of $10 \mathrm{mM} \mathrm{HAuCl}_{4}$ and $65 \mu \mathrm{L}$ of $100 \mathrm{mM}$ L-ascorbic acid solutions. Afterwards, $250 \mu \mathrm{L}$ of the above seed solution was added and the reaction mixture was left undisturbed for $30 \mathrm{~min}$. The Au octahedra were purified in a similar manner as described above for nanocubes. 


\subsection{Size Distribution Characterization of Au Nanocrystals}

The synthesized nanocrystals, Au nanocube and Au octahedra were characterized its size distribution.
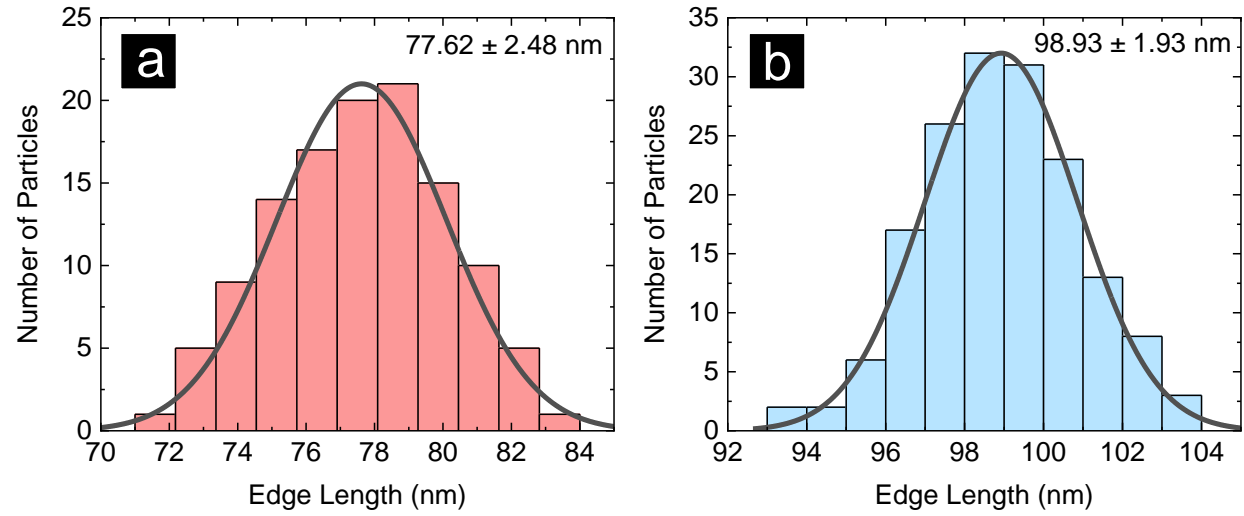

Figure S2. Size distribution for the edge length of Au NC (a) and Au OD (b). The average edge length for Au OD and Au NC are ca. $99 \mathrm{~nm}$ and $78 \mathrm{~nm}$, respectively.

Table S1. Synthesized nanocrystals dimension estimation of Au nanocube and Au octahedra.

\begin{tabular}{|c|c|c|}
\hline & Au NC & Au OD \\
\hline Edge length $(\mathbf{n m})$ & $77.55( \pm 2.39)$ & $98.93( \pm 1.93)$ \\
\hline Surface area $\left(\mathbf{n m}^{\mathbf{2}}\right)$ & 30070.0 & 29665.7 \\
\hline Volume $\left(\mathbf{n m}^{\mathbf{3}}\right)$ & 466385.9 & 456433.8 \\
\hline
\end{tabular}



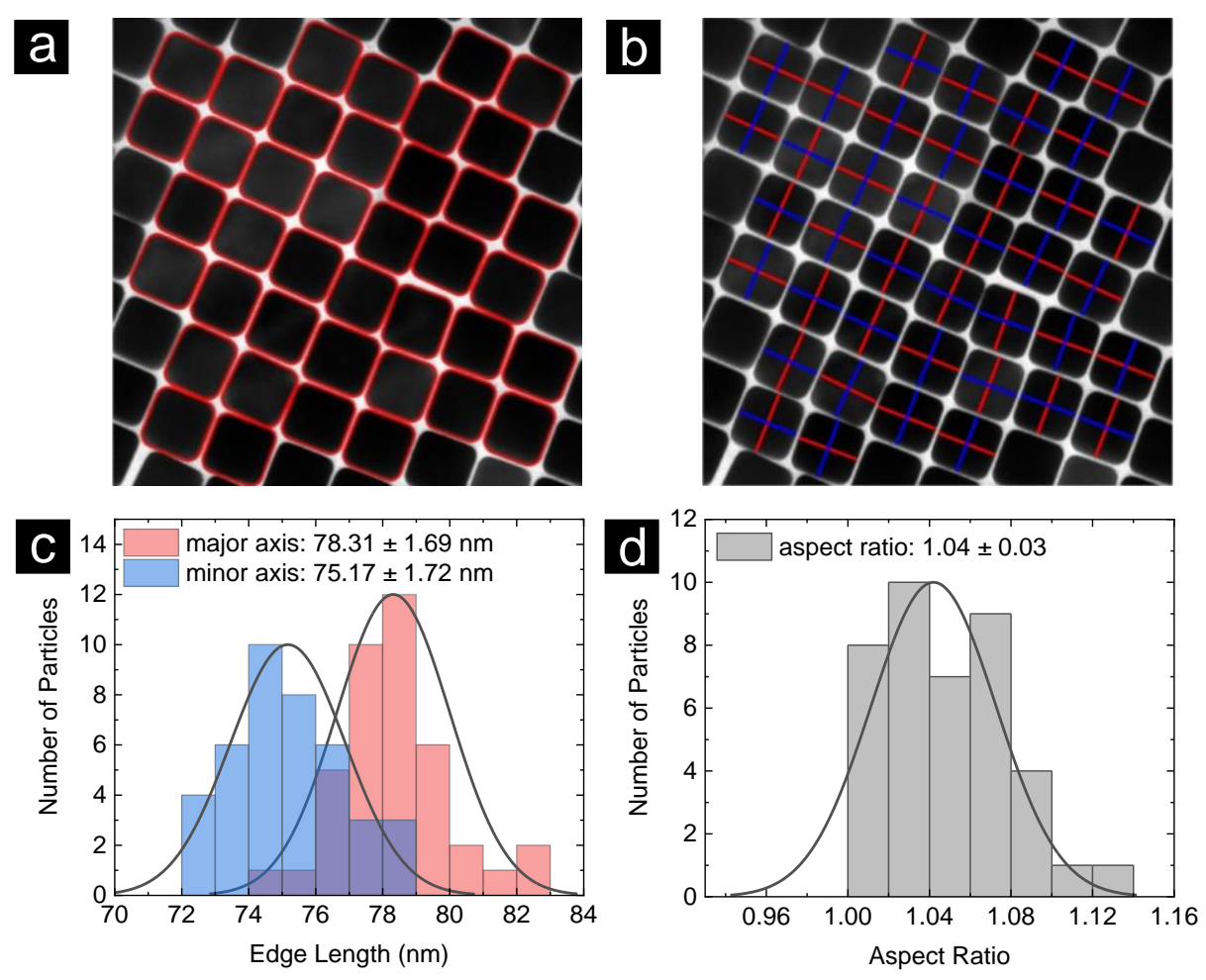

Figure S3. Representative Matlab calculation for the length of major and minor axis of Au NCs from TEM images. Each Au NC in the image were recognized as single entity by fitting contour (a). In the measured contour, the longest axis was measured as the major axis, and the perpendicular axis was collected as the minor axis (b). The average major and minor axis length were ca. 78 and $75 \mathrm{~nm}$, respectively (c). Aspect ratio of each Au NC was calculated by dividing the measured major by the minor axis (d). The aspect ratio was ca. 1.04, which showed that the $\mathrm{Au}$ NCs were mostly cubic structure. 


\section{Section S3. Preparation of Working Electrodes}

\subsection{Cleaning of Glassy Carbon Substrates}

Glassy carbon plates $(1.2 \mathrm{~cm}$ x $2.4 \mathrm{~cm}, 99.99 \%$, Alfa Aesar) were thoroughly cleaned prior to the deposition of Au nanoparticles. Typically, a glassy carbon substrate was soaked in a freshly prepared aqua regia solution for $1 \mathrm{~min}$ to remove metal impurities, followed by mechanical polishing with alumina powder ( $\mathrm{CH}$ Instrument) in the order of 1.0, 0.5, and $0.05 \mu \mathrm{m}$ particle size. Afterwards, the polished substrate was sonicated in a 1:1 (v:v) mixture of water and isopropanol followed by acetone for $1 \mathrm{~min}$. each. After drying with $\mathrm{N}_{2}$, the glassy carbon substrate was polished against a wet polishing pad to remove residual alumina particles. Finally, the substrate was sonicated in the same manner as described above and dried with $\mathrm{N}_{2}$. SEM was used to inspect the substrates to ensure that they are free of alumina particles. (Figure S4).

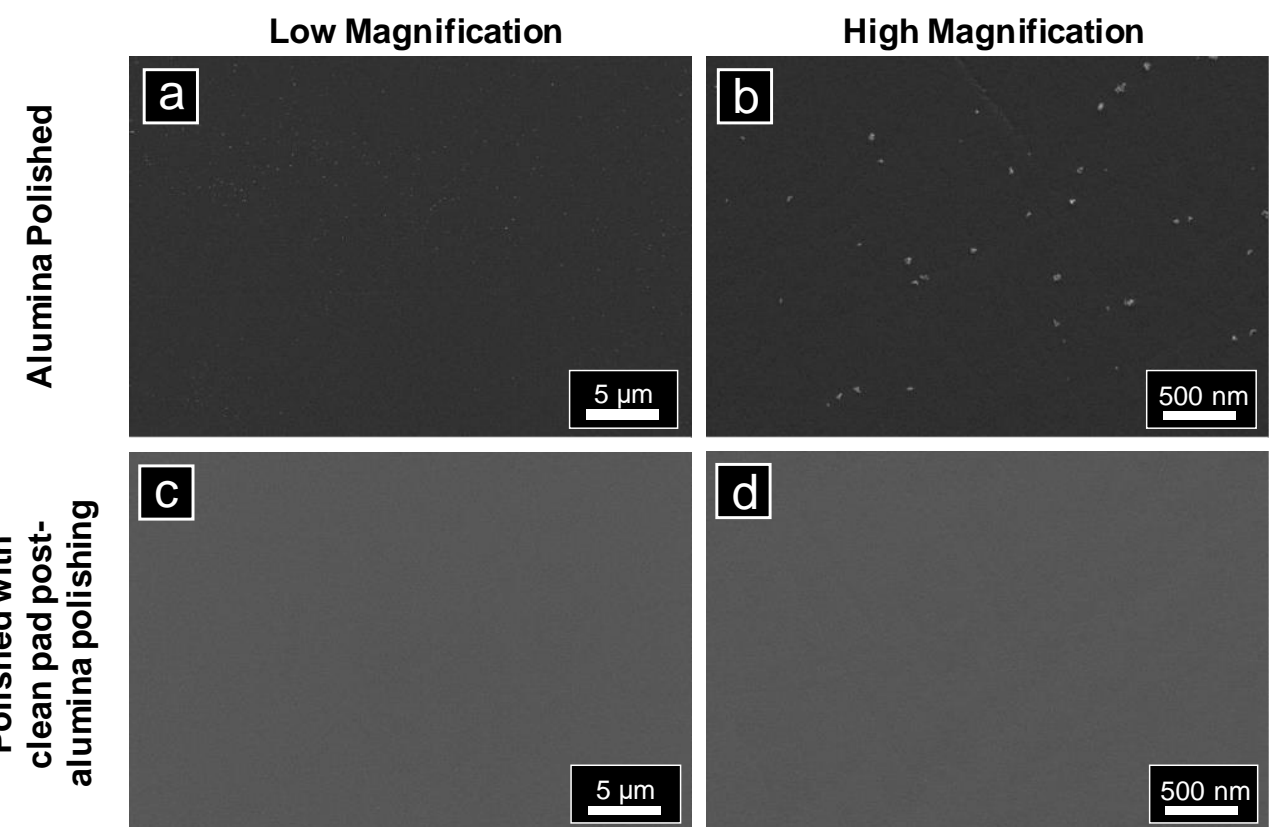

Figure S4. SEM images of glassy carbon substrates $(a, b)$ after polishing with alumina slurry in the order of $1.0,0.5$, and $0.05 \mu \mathrm{m}$ particle diameters and (c,d) after additional polishing against a wet polishing pad. 


\subsection{Preparation of Au NPs Drop-casted Glassy Carbon Electrodes}

SECCM measurements works best on glassy carbon substrates sparsely covered with nanoparticles without the formation of extended nanoparticle aggregates. To ensure that the loadings of $\mathrm{Au}$ NCs and ODs were identical, the concentration of NP solution was determined by Beer's law $(A=\varepsilon b c)$ through UV-Vis spectroscopy. Since optical path length $(b)$ was fixed and the extinction coefficient $(\varepsilon)$ of $78 \mathrm{~nm} \mathrm{Au} \mathrm{NCs} \mathrm{and} 99 \mathrm{~nm}$ ODs were known, ${ }^{2}$ peak absorbance measured from UV-Vis spectrum was used to equalize the particle number concentration (c). 15 $\mu \mathrm{L}$ of Au nanoparticle solution was drop-casted onto a glassy carbon substrate (Figure S5).
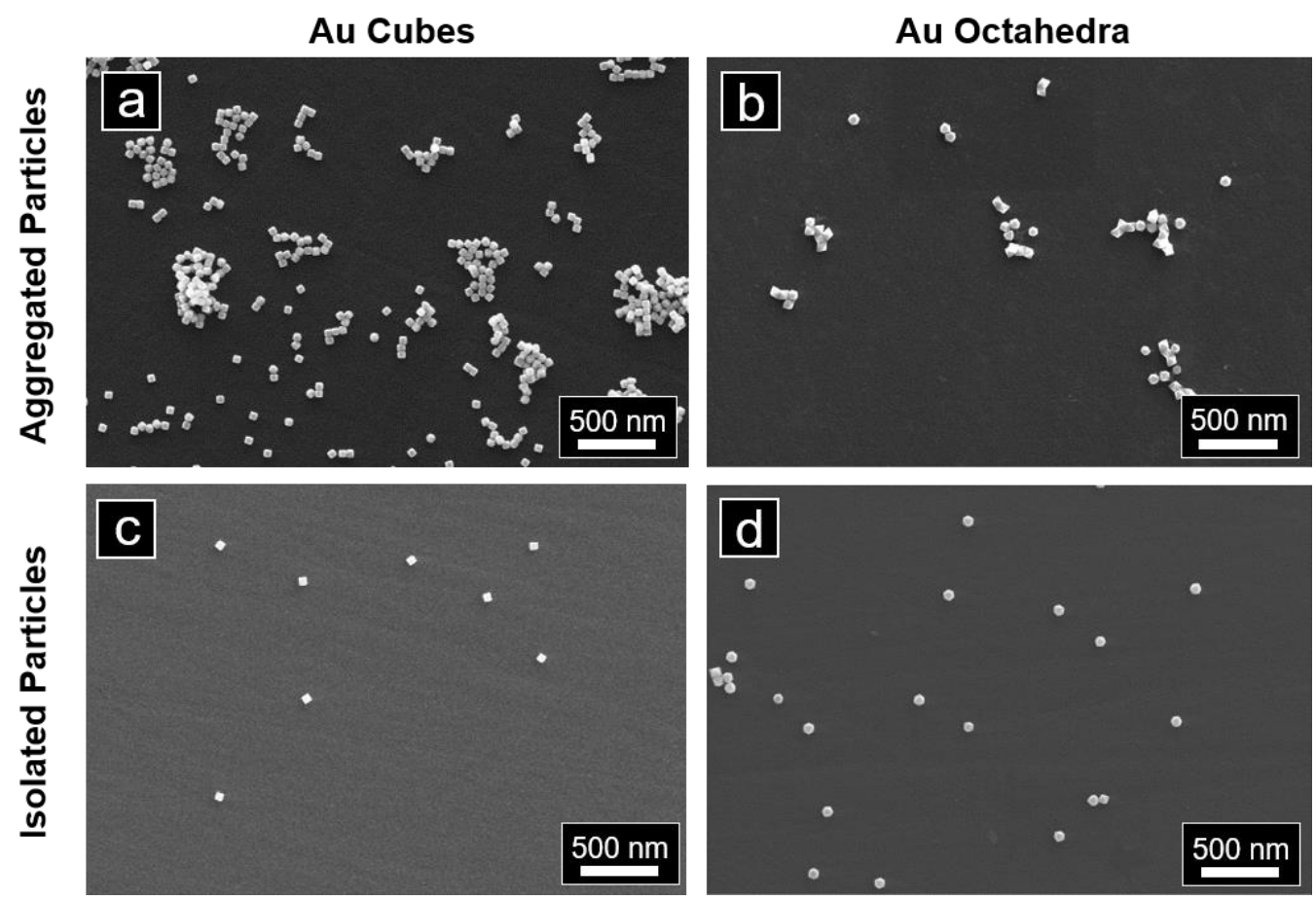

Figure S5. SEM images of glass carbon electrodes covered with Au nanoparticles. (a,b) Cubes and octahedra deposited from solutions with O.D. $=2 .(\mathrm{c}, \mathrm{d})$ Cubes and octahedra deposited from solutions with (c) O.D. $=0.67$ (cubes) and (d) O.D. $=0.37$ (octahedra). 


\subsection{Post-Deposition Removal of CTAB Ligands}

Herein, we developed a two-step process to remove free $\mathrm{CTAB}$ surfactants and bound CTAB ligands on the nanoparticle surface. In a typical workflow, glassy carbon substrates coated with $\mathrm{Au}$ nanoparticles were first immersed in methanol for about 2 minutes to remove free $\mathrm{CTAB}$ surfactants and a significant fraction of CTAB ligands due to its high solubility in alcohols (solubility of CTAB in $\mathrm{H}_{2} \mathrm{O}: 36.4 \mathrm{mg} / \mathrm{mL}$ at $20^{\circ} \mathrm{C}$; solution of $\mathrm{CTAB}$ in methanol: $>200 \mathrm{mg} / \mathrm{mL}$ at $20^{\circ} \mathrm{C}$ ). The methanol treatment duration was optimized by monitoring the intensity of symmetric $\left(\sim 2849 \mathrm{~cm}^{-1}\right)$ and asymmetric $\left(\sim 2914 \mathrm{~cm}^{-1}\right)$ C-H stretching vibrations using FTIR spectroscopy (Figure S6). Usually after 2-min of methanol treatment, $\mathrm{C}-\mathrm{H}$ vibrations were no longer visible on the FTIR spectrum. However, due to the detection limit of FTIR, a trace amount of CTAB and hydrocarbon impurities can still remain, as illustrated by the SEM images shown in Figure S7 and

\section{Figure S8.}

Cyclic voltammetry $(\mathrm{CV})$ was performed to further "clean" the nanoparticles and the glassy carbon substrate. Electrochemical experiments were carried out with a custom-built threeelectrode setup (Figure S9). A glassy carbon electrode was used as the working electrode, platinum gauze (52 mesh woven from $0.1 \mathrm{~mm}$ diameter Pt wire, $99.9 \%$, Alfa Aesar) as the counter electrode and $\mathrm{Ag} / \mathrm{AgCl}(3.5 \mathrm{M} \mathrm{NaCl}, \mathrm{BASi})$ as the reference electrode. $100 \mathrm{mM} \mathrm{HClO}_{4}(70 \%$ in water, Acros Organics) was used as the electrolyte. The working electrode was enclosed within a Teflon box with a precision-machined front opening to define the working electrode area and to reduce background interference.

Electrochemical measurements were carried out by using a potentiostat (SP-300, Biologic). The working electrode potential was swept from $0 \mathrm{~V}$ to $-1.0 \mathrm{~V}$ vs. $\mathrm{Ag} / \mathrm{AgCl}(3.5 \mathrm{M} \mathrm{NaCl})$ at the scanning rate of $100 \mathrm{mV} / \mathrm{s}$ for 2 cycles (data is not shown). SEM imaging revealed that the size 
and morphology of $\mathrm{Au}$ nanoparticles were preserved after these $\mathrm{CV}$ scans, yet the amount of surface contamination likely caused by residual CTAB was dramatically reduced according to results from SEM imaging (Figure S7,S8). Specifically, the white deposits on bare glassy carbon areas (Figure S7a and S8a) and the white "halo" surrounding individual nanoparticles (Figure S7b and S8b) were observed to diminish substantially upon soaking in methanol (Figure S7c,d and S8c,d) and CV treatment (Figure S7e,f and S8e,f).
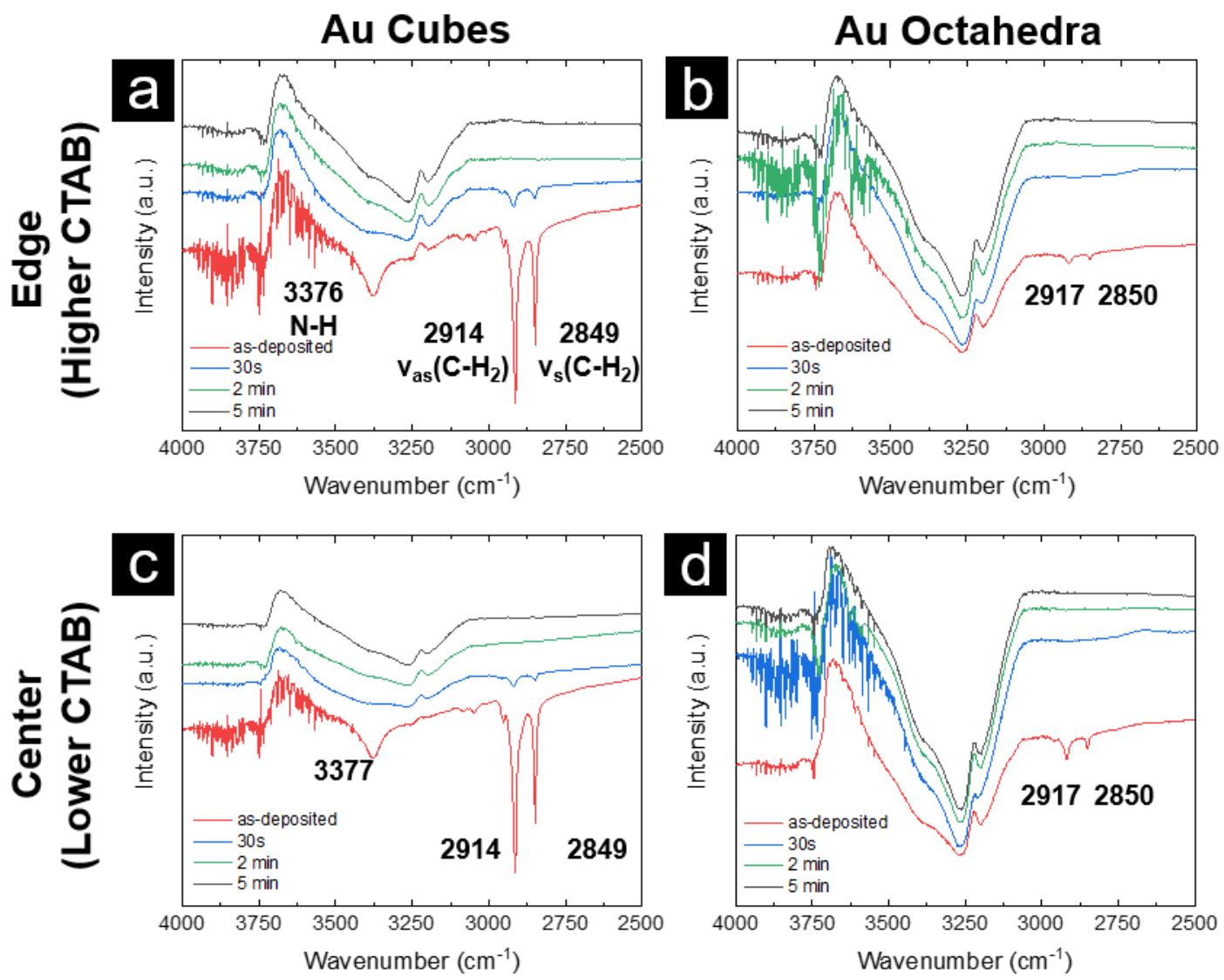

Figure S6. FTIR spectra of glass carbon electrodes coated with Au nanoparticles (red) and after soaking in methanol for 30 seconds (blue), 2 min (green) and 5 min (black). FTIR spectra acquired from $(a, b)$ the periphery of nanoparticle-coated area showed higher $\mathrm{C}-\mathrm{H}$ vibrational signals than those from $(\mathrm{c}, \mathrm{d})$ center of nanoparticle deposits for both cubes $(\mathrm{a}, \mathrm{c})$ and octahedra $(b, d)$. 

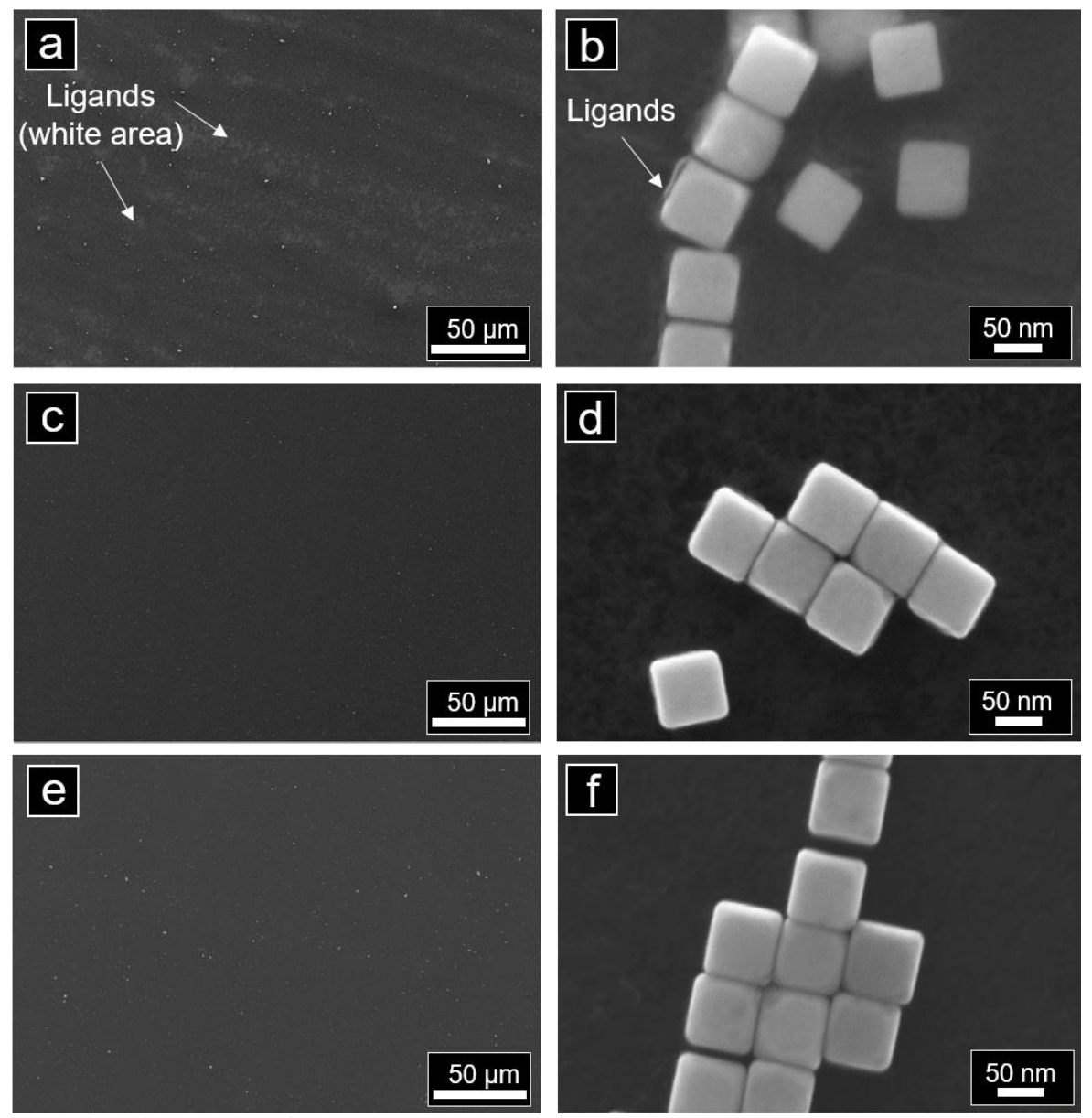

Figure S7. SEM images of glassy carbon substrates coated with Au nanocubes(a,b) Thick deposits on the substrate (a) and white halos (b) around individual nanocubes were observed, both of which are likely due to residual CTAB. The amount of CTAB was significantly reduced after soaking in methanol $(\mathrm{c}, \mathrm{d})$ followed by $\mathrm{CV}$ treatment $(\mathrm{e}, \mathrm{f})$. 

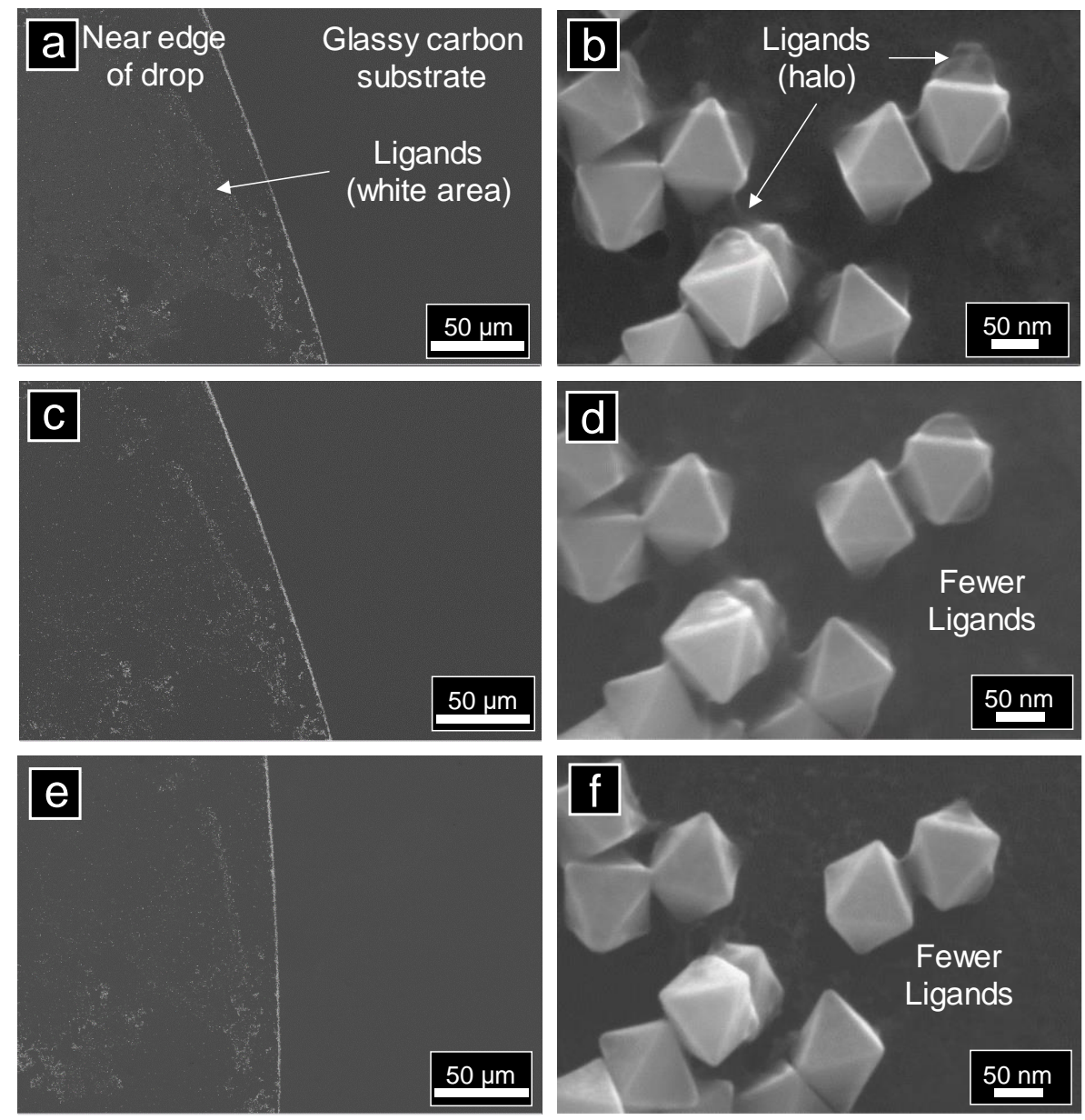

Figure S8. SEM images of glassy carbon substrates coated with Au octahedra. (a,b) Thick deposits on the substrate (a) and white halos around individual octahedra were observed (b), both of which are likely caused by residual CTAB. The amount of CTAB was significantly reduced after soaking in methanol $(c, d)$ followed by $\mathrm{CV}$ treatment $(\mathrm{e}, \mathrm{f})$. 


\subsection{Macroscale Linear Sweep Voltammetry}

Samples for macroscale electrochemical measurements were prepared as follows: The glassy carbon was placed in a custom-made Teflon box to ensure that the geometric area of electrode was consistent for all macroscale voltammetry studies. Then, $15 \mu \mathrm{L}$ of Au NCs or OD was drop-cast onto the glassy carbon area defined by the circular hole on one face of the Teflon box. Nanoparticle number concentrations were kept the same between NC and OD solutions, as detailed in Section

3.2 .
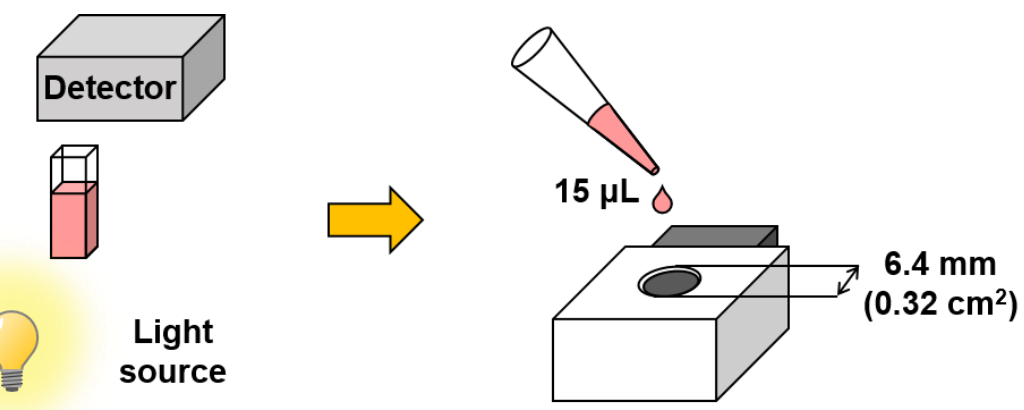

Step 1:

Concentration control by UV-Vis spectroscopy

Step 2:

Drop-casting and drying on fixed surface area

Figure S9. Schematic workflow for preparation of electrodes with equal particle concentration. 

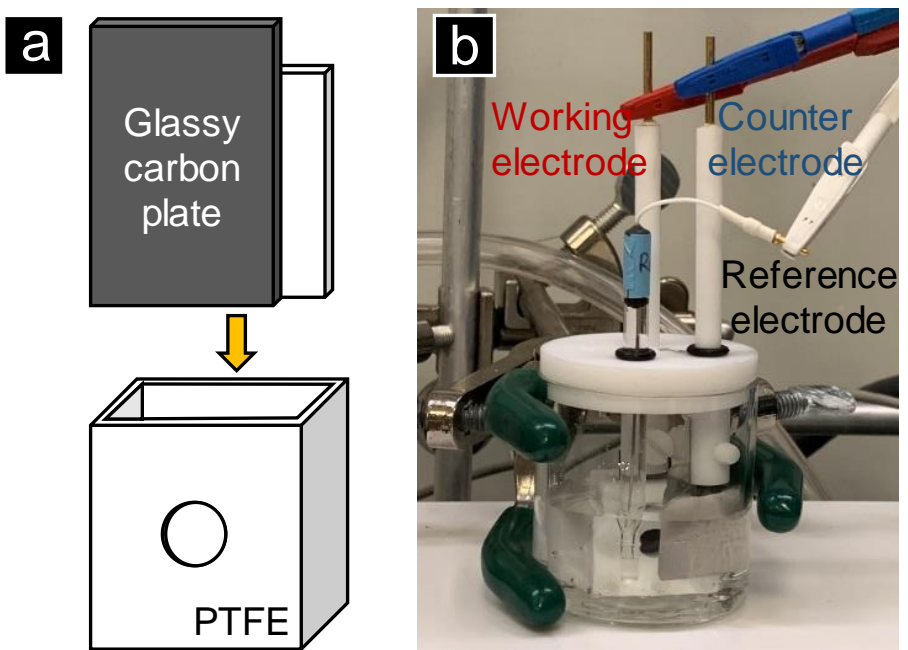

Figure S10. (a) Schematic of the glassy carbon working electrode and the Teflon box. (b) A photograph of the actual three-electrode electrochemistry setup. 


\subsection{IR-Drop Compensation}

Uncompensated resistance $\left(\mathrm{R}_{\mathrm{u}}\right)$ was determined by using potentiometric electrochemical impedance spectroscopy (PEIS) according to a method reported previously with modifications. ${ }^{4}$ Specifically, $20 \mathrm{mV}$ of sinusoidal AC waveforms, centered at the open-circuit potential, were applied with frequencies ranging from $1 \mathrm{~Hz}$ to $7 \mathrm{MHz}$ with 10 frequencies measured per decade. A Bode plot was constructed to define characteristic frequency and $R_{u}$, which were typically $\sim 100$ $\mathrm{kHz}$ and $\sim 80 \Omega$, respectively for a bare glassy carbon electrode (Figure S11a). The $\mathrm{R}_{\mathrm{u}}$ value was consistent with that determined from a series of Nyquist plots at different potentials (Figure S11b). A glassy carbon electrode covered with Au NCs or ODs showed a typical $\mathrm{R}_{\mathrm{u}}$ of $\sim 80 \Omega$, whereas a
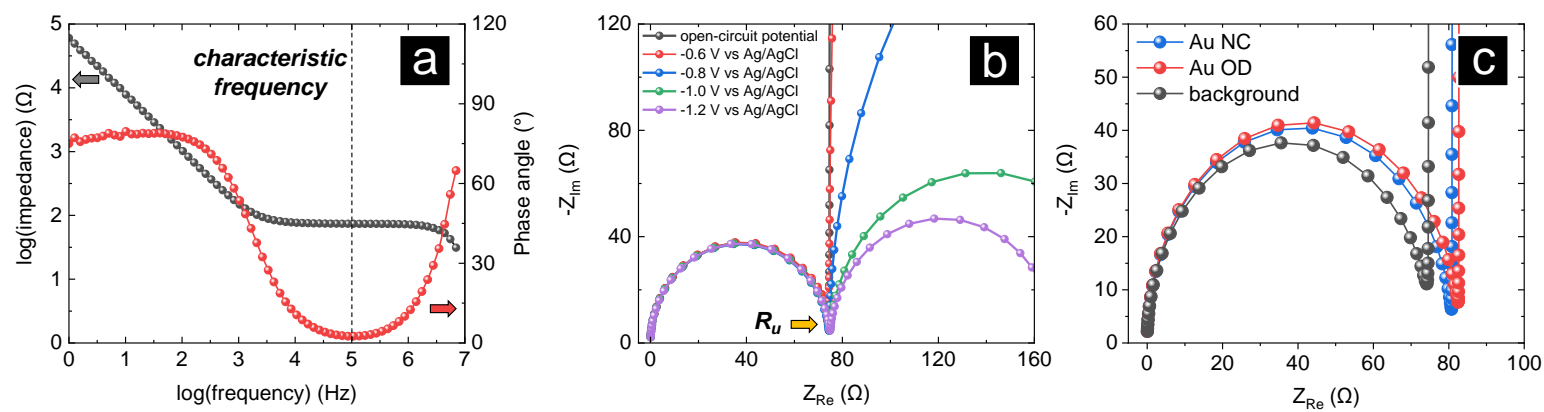

Figure S11. Determination of uncompensated resistance $\left(R_{u}\right)$ used for IR-drop compensation using PEIS. (a) Bode plot was constructed to measure characteristic frequency and $\mathrm{R}_{\mathrm{u}}$.at bare glassy carbon. (b) Nyquist plots generated at different applied potentials exhibited $\mathrm{R}_{\mathrm{u}}$ at bare glassy carbon. (c) Representative Nyquist plots for glassy carbon electrodes coated with Au NCs, ODs, and bare glassy carbon electrodes. 
bare glassy carbon electrode exhibited a $R_{u}$ of $\sim 70 \Omega$ (Figure S11c). IR-drop correction was performed by using the following equation:

$$
V_{I R-\text { corrected }}=V_{\text {Measured }}-\text { average current }(A) \times R_{u}(\Omega)
$$

IR-drop compensation was not carried out for SECCM raw data given that the IR-drop was negligible in these measurements.
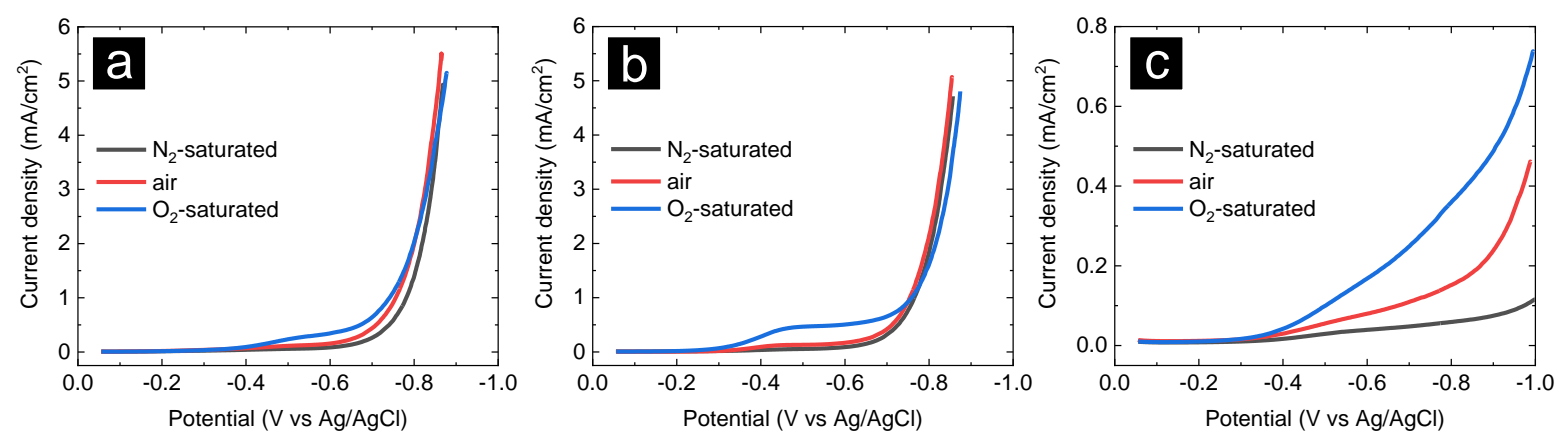

Figure S12. Macroscale LSVs at Au NC (a), Au OD (b), and background glassy carbon (c) when the atmosphere of electrochemical system was saturated by nitrogen (black), air (red), and oxygen (blue). The electrolyte was aqueous $100 \mathrm{mM} \mathrm{HClO}_{4}$ and the scan rate was $50 \mathrm{mV} / \mathrm{s}$. Potential value was corrected by uncompensated resistance which was measured via electrochemical impedance spectroscopy. 


\subsection{Calibration of the Ag/AgCl Reference Electrode}

The $\mathrm{Ag} / \mathrm{AgCl}$ reference electrode (3.5 $\mathrm{M} \mathrm{NaCl}$, BASi) was utilized for bulk measurement and $\mathrm{Ag} / \mathrm{AgCl}$ wire quasi-reference electrode was used for SECCM measurement. The $\mathrm{Ag} / \mathrm{AgCl}$ wire electrode was calibrated by measuring open-circuit potential against the $\mathrm{Ag} / \mathrm{AgCl}$ electrode on a daily basis prior to any electrochemical measurements. For calibration, the $\mathrm{Ag} / \mathrm{AgCl}$ electrode and the $\mathrm{Ag} / \mathrm{AgCl}$ wire electrode were placed inside a one-compartment electrochemical cell filled with $100 \mathrm{mM} \mathrm{HClO}_{4}$ electrolyte. Open-circuit potential between the two electrodes was measured by a potentiostat, which gives the potential value correction factor for calibrating $\mathrm{Ag} / \mathrm{AgCl}$ wire electrode. Experimental potential values versus the $\mathrm{Ag} / \mathrm{AgCl}$ reference electrode for SECCM measurement were determined as follows:

$$
E_{v s \mathrm{Ag} / \mathrm{AgCl}(3 \mathrm{M} \mathrm{NaCl})}=E_{v s \mathrm{Ag} / \mathrm{AgCl} \text { wire }}+E_{\text {open-circuit potential correction factor }}
$$

\subsection{Instrumentation}

SEM images were collected on a Carl Zeiss Auriga FIB-SEM. FTIR spectra were recorded on a Bruker Vertex 70V FT-IR spectrometer in transmission mode. 


\section{Section S4. Scanning Electrochemical Cell Microscopy (SECCM)}

\section{Instrumentation and Operation}

\subsection{Chemicals and Materials}

All solutions were prepared with ultrapure $\mathrm{H}_{2} \mathrm{O}$ at $25{ }^{\circ} \mathrm{C}$ from a Milli-Q water purification system (18.2 M $\Omega \cdot \mathrm{cm}$, Millipore Corp.). Potassium chloride (KCl, VWR Analytical), potassium perchlorate $\left(\mathrm{KClO}_{4}\right.$, Mallinckrodt), and perchloric acid $\left(\mathrm{HClO}_{4}\right.$, Fisher Scientific) were used as received. Glassy carbon (Alfa Aesar) were used as substrates. A metathesis reaction was used to convert (ferrocenylmethyl) trimethylammonium iodide (FcTMA ${ }^{+} \mathrm{I}^{-}$, Strem Chemicals) to (ferrocenylmethyl) trimethylammonium hexafluorophosphate (FCTMA $\left.{ }^{+} \mathrm{PF}_{6}^{-}\right)$. The resulting hexafluorophosphate salt was dried and stored at room temperature prior to use. A home-built humidity chamber was used to keep the humidity (ca. 20 40 \%) stable SECCM CV map operation and measurement.

\subsection{Fabrication of Nanopipettes for SECCM}

Theta barrel quartz capillaries (O.D. $1.2 \mathrm{~mm}$, I.D. $0.9 \mathrm{~mm}$, QT120-90-7.5, Sutter Instrument, Novato, CA) were pulled into nanopipettes with inner and outer diameters of $50-70 \mathrm{~nm}$ and 150 $-200 \mathrm{~nm}$, respectively, with a laser puller equipped with a $\mathrm{CO}_{2}$ laser (P-2000, Sutter Instrument). The following laser pulling program was used: Heat: 700, Filament: 3, Velocity: 35, Delay: 150, Pull: 150. Pipette dimensions were characterized by SEM (FEI Quanta 600F). The pipettes were filled with $100 \mathrm{mM} \mathrm{HClO}_{4}, 2 \mathrm{mM} \mathrm{FcTMA}^{+} \mathrm{PF}_{6}{ }^{-}$and $50 \mathrm{mM} \mathrm{KCl}$, or $2 \mathrm{mM} \mathrm{HClO}_{4}$ and $10 \mathrm{mM}$ $\mathrm{KClO}_{4}$. Two $\mathrm{Ag} / \mathrm{AgCl}$ wires were inserted into each barrel of the pipette to serve as quasi-reference counter electrodes (QRCEs). Ag/AgCl wires were prepared from perfluoroalkoxy (PFA, A-M 
Systems, Sequim, WA) coated silver wire that were stripped to reveal the bare silver wire and subsequently chloridized. $\mathrm{Ag} / \mathrm{AgCl} \mathrm{QRCEs}$ in perchloric acid were previously reported to show a stable reference potential with a drift in potential of about $1 \mathrm{mV}$ per hour. ${ }^{5}$ All SECCM voltammetric data was calibrated to a $\mathrm{Ag} / \mathrm{AgCl}(3.5 \mathrm{M} \mathrm{NaCl})$ reference electrode.

\subsection{SECCM Instrumentation and Operation of SECCM}
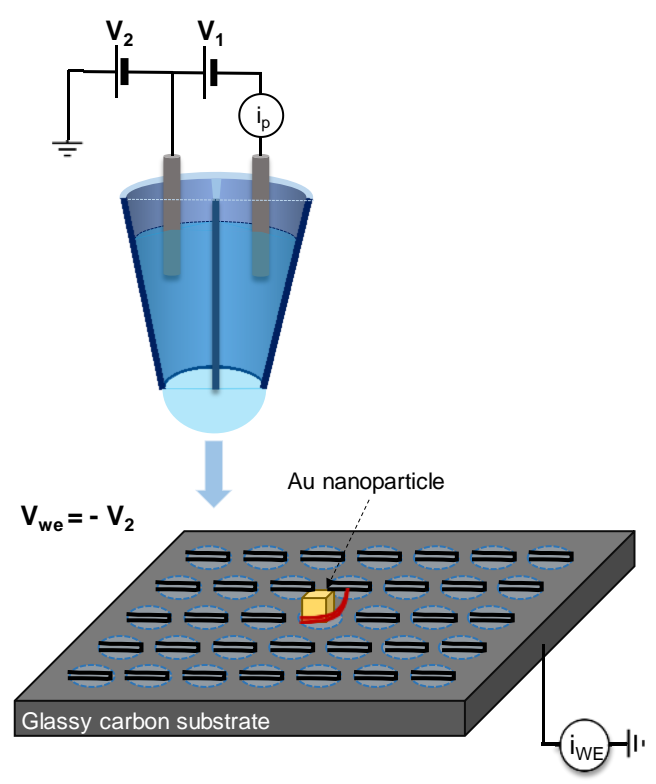

Figure S13. Schematic of the scanning electrochemical cell microscopy (SECCM) setup with $\mathrm{Au}$ nanoparticle deposited on the glassy carbon. The current signal generated from heterogenous electrocatalytic reaction is recorded through ammeter (current amplifier) connected to the conductive glassy carbon substrate when SECCM probe reaches the position of Au nanoparticle during CV mapping.

To operate SECCM, a specifically designed home-built potentiostat was interfaced to a commercial AFM/SICM system (XE-Bio, Park Systems, South Korea). The working principles for the SECCM instrumentation were detailed in previous publications from the Unwin group. ${ }^{6-7}$ 
The home-built potentiostat allows SECCM to apply and to control the desired electrical potential at glassy carbon (GC) where a small electrolyte meniscus at the tip-end of SECCM probe contacts the surface of WE. The schematic of SECCM setup is shown in Figure S13. All SECCM measurements were carried out in the ambient environment (i.e. oxygen was present in drop and the gaseous environment surrounding the drop).

The electrolyte meniscus serves as a small electrochemical cell in SECCM. The meniscus size is in the range of attoliters by considering the probe-tip dimension (dual barrel pipette outer diameter 150-250 nm, Figure S14).
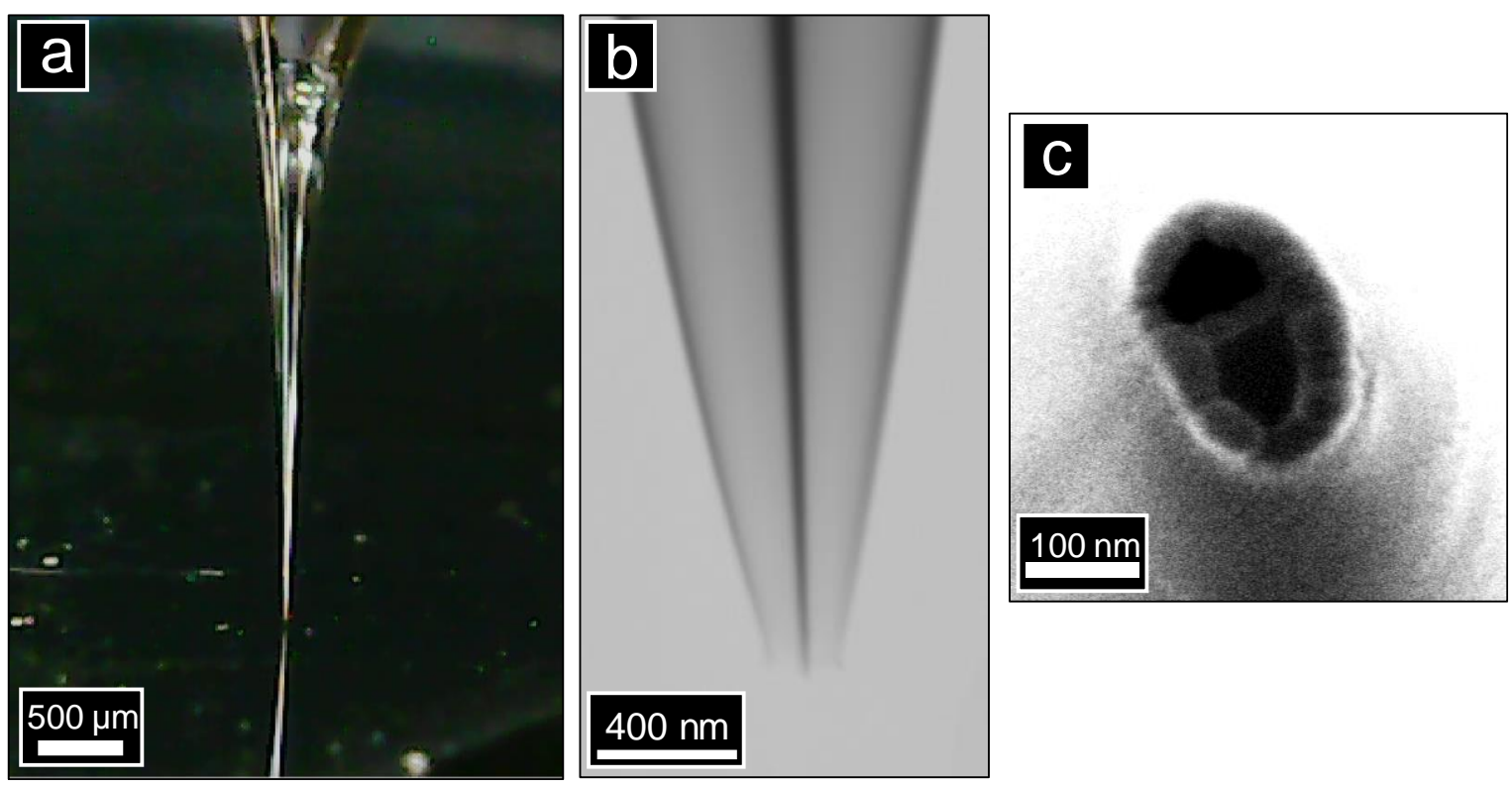

Figure S14. (a) An optical micrograph of SECCM probe engaged on the glassy carbon surface (collected by high resolution digital microscope). (b) Scanning transmission electron image of dual barrel SECCM probe-tip and (c) SEM image of the tip of a dual barrel pipette that was coated with ca. $5 \mathrm{~nm} \mathrm{Au} / \mathrm{Pd}$. 

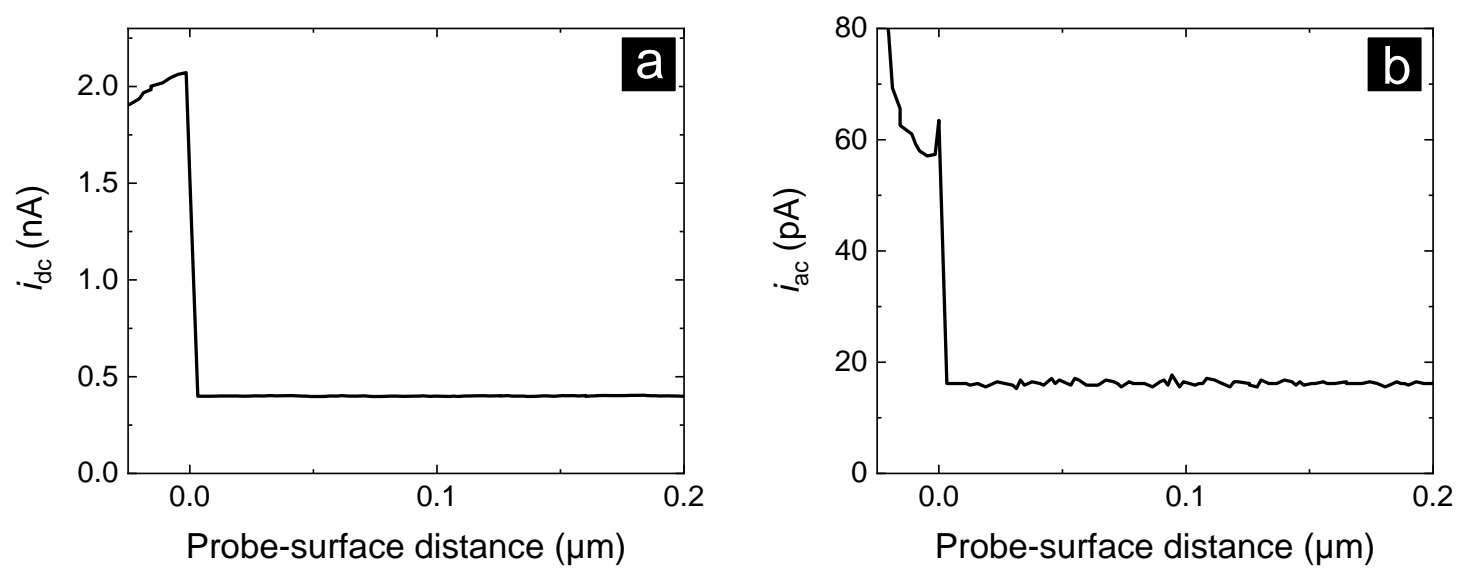

Figure S15. SECCM approach curves with a dual barrel pipette, filled with $100 \mathrm{mM} \mathrm{HClO}$. (a) dc component and b) ac component of ion current as a function of probe-surface distance. The initial $\mathrm{i}_{\mathrm{dc}}$ and $\mathrm{i}_{\mathrm{ac}}$ were $0.4 \mathrm{nA}$ and $16 \mathrm{pA}$ respectively which increase to $2 \mathrm{nA}$ and $60 \mathrm{pA}$ when the meniscus contacted the surface of the bare glassy carbon substrate. The probe-surface distance was adjusted by the $\mathrm{Z}$ detector signal ( $\mathrm{Z}$ piezo-scanner elongation, directly detected by a string gauge, attached on the piezo). The zero point for the probe-surface distance was defined as the position where $i_{d c}$ and $i_{a c}$ began to increase in magnitude. The velocity of probe approach was $100 \mathrm{~nm} \mathrm{~s}^{-1}$.

For safe meniscus contact in repeat to substrate (working electrode), ionic current in DC and AC components were carefully monitored as figure S15. Once the meniscus contacts the GC substrate, the potential $\left(\mathrm{V}_{2}\right)$ sweep (ca.in the range of 0 to $1.0 \mathrm{~V}$ ) is carried out to acquire cyclic voltammogram while the potential $\left(\mathrm{V}_{1}\right)$, the difference between $\mathrm{Ag} / \mathrm{AgCl}$ wires in each barrel of the pipette is kept constant $(100 \mathrm{mV})$. In the home-built potentiostat, the potential difference is actually formed by applying $\pm \mathrm{V}_{1} / 2$ onto two $\mathrm{Ag} / \mathrm{AgCl}$ electrodes. For example, one electrode as 
$-50 \mathrm{mV}$ and the other as $+50 \mathrm{mV}$ to generate $100 \mathrm{mV}$ potential difference. Meanwhile, the potential sweep that is applied to $\mathrm{V}_{2}$ changes both $\mathrm{Ag} / \mathrm{AgCl}$ electrodes simultaneously. Therefore, the effective potential $\left(\mathrm{V}_{\text {we }}\right)$ at $\mathrm{GC}$ substrate with respect to the $\mathrm{Ag} / \mathrm{AgCl}$ electrodes is approximately $-V_{2}$. The electrochemical current signal generated at the working electrode $\left(i_{w e}\right)$ is recorded by a current amplifier (CHEM-CLAMP, Dagan Corp., U.S.A.) equipped with a $100 \mathrm{M} \Omega$ headstage. In Figure S16, the $\mathrm{E}_{1 / 2}$ recorded for the $\mathrm{FcTMA}^{+} / \mathrm{FcTMA}^{2+}$ redox couple collected using macroscale cyclic voltammetry vs a nanoscale CV collected with SECCM were compared in a solution containing $2 \mathrm{mM} \mathrm{FcTMA}^{+}$and $50 \mathrm{mM} \mathrm{KCl}$.
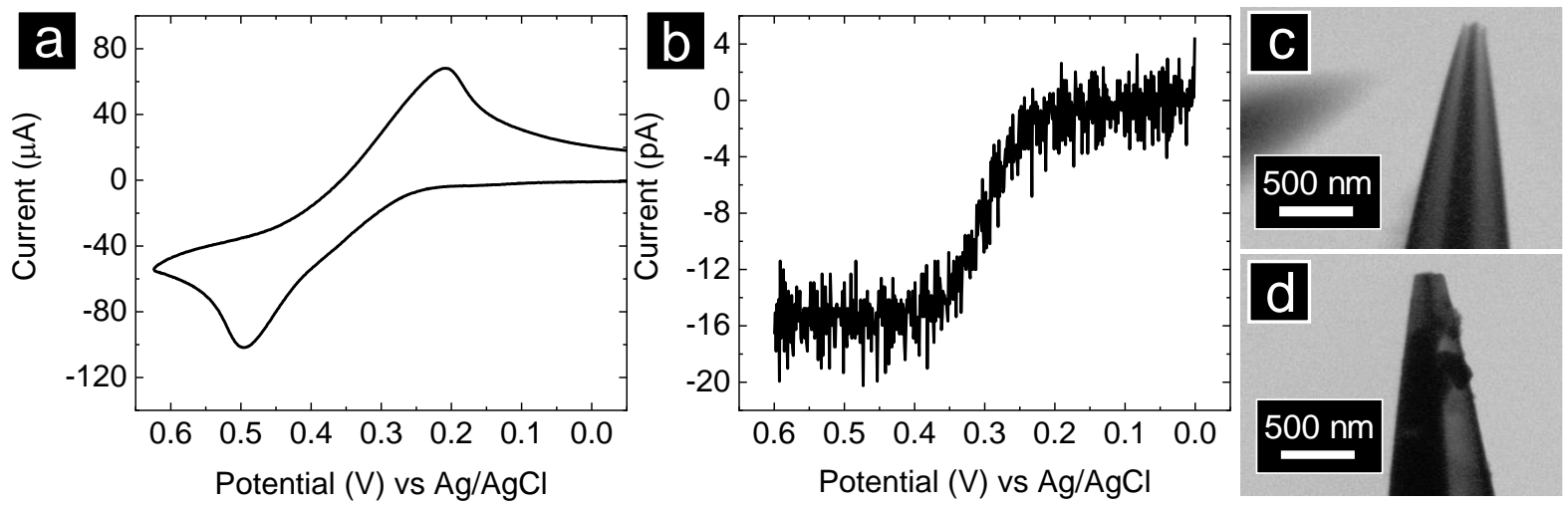

Figure S16. Cyclic voltammograms collected in a solution containing $2 \mathrm{mM} \mathrm{FcTMA}^{+}$and 50 $\mathrm{mM} \mathrm{KCl}$ at a scan rate of $1.2 \mathrm{~V} / \mathrm{s}$ (a) in bulk at a $3 \mathrm{~mm}$ glassy carbon electrode and (b) at the nanoscale by SECCM CV mapping at a glassy carbon substrate. $\mathrm{E}_{1 / 2}$ in (a) and (b) were 0.38 $\mathrm{V}$ and $0.30 \mathrm{~V}$ vs $\mathrm{Ag} / \mathrm{AgCl}$, respectively. STEM images of the pipette (c) before and (d) after SECCM CV mapping. The outer diameter of the pipette in (c) and (d) were ca. $200 \mathrm{~nm}$, which indicates that the pipette did not break during CV mapping.

One of major issues with constructing a new SECCM setup on the platform of a commercial instrument was signal-to-noise ratio. The Unwin Group also carefully considered the signal-to- 
noise ratio of their instrument. ${ }^{8}$ This issue is closely related with the tip dimension of SECCM probes because the smaller probe-tip size (typical outer diameter: $150 \sim 250 \mathrm{~nm}$ for probes used here), the lower the signal and signal-to-noise ratio. For the SECCM setup used here, peak-to-peak RMS noise is 2 3 pA (data not shown), which was at least an order of magnitude lower than HER/ORR currents achieved at individual pixels. The repeatability of CVs was also evaluated by comparison of the tip dimension of SECCM probe before and after CV map completion with 312 local position of bare glassy carbon (Figure S17).
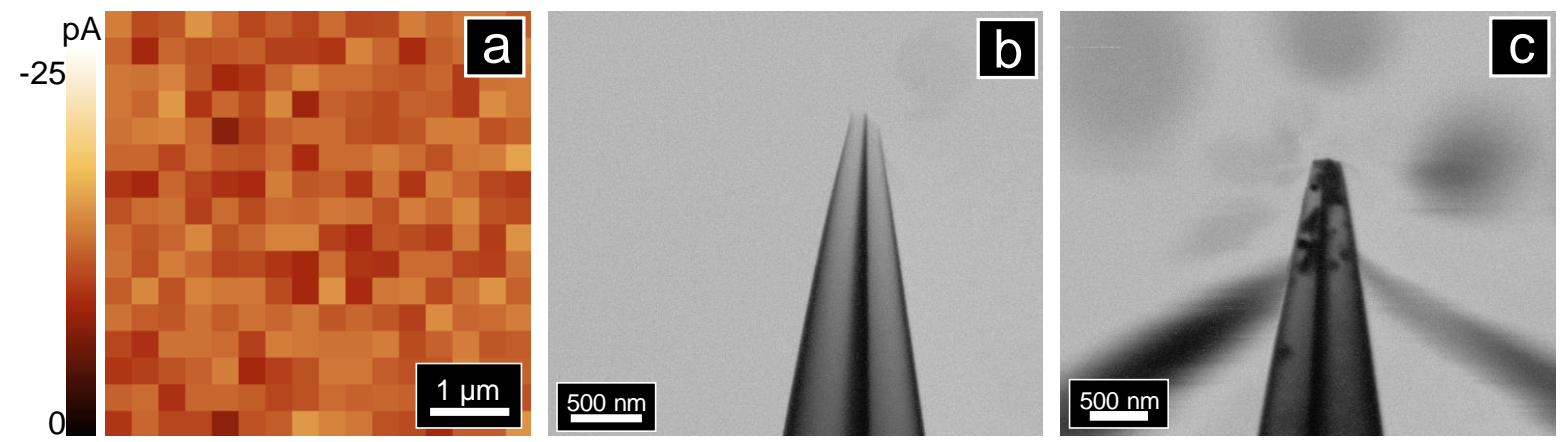

Figure S17. Cyclic voltammetry repeatability evaluation for SECCM CV mapping. (a) SECCM CV map collected in a solution containing $2 \mathrm{mM} \mathrm{FcTMA}^{+}$and $50 \mathrm{mM} \mathrm{KCl}$ at effective potential of $360 \mathrm{mV}$ vs $\mathrm{Ag} / \mathrm{AgCl}$ (at where limiting current of redox mediator appeared) with a scan rate of $1.2 \mathrm{~V} / \mathrm{s}$ on the surface of bare (polished) glassy carbon electrode surface. Scan size was $4 \mu \mathrm{m} \times 4 \mu \mathrm{m}$ with total 312 individual CVs ( $16 \times 16$ pixels). (b) electron micrograph of the sister pipette of the one used for this SECCM CV map with $223 \mathrm{~nm}$ outer diameter (represent the dimension of probe-tip before SECCM CV mapping). (c) electron micrograph of the dual barrel pipette tip used for this SECCM CV map with $252 \mathrm{~nm}$ outer diameter (represent the dimension of probe-tip after SECCM CV mapping). The dimension of SECCM probe-tip didn't change much (only about $\sim 20 \mathrm{~nm}$ ) during 312 individual CVs performance. 
The SECCM probe was engaged at the GC surface under AC (alternating current) mode ${ }^{7,9}$ (physical modulation $\sim 10 \mathrm{~nm}$ ) feedback control to prevent physical contact or damage of probeend which allows consistent meniscus contact status during potential sweep and the acquisition of CVs. For the acquisition of one CV map with SECCM, the pixel resolution was optimized to maximize the detection rate of nanoparticles. A pixel resolution of $200 \mathrm{~nm} /$ pixel was used for $\mathrm{Au}$ NCs (edge length: $\sim 78 \mathrm{~nm}$ ) Au ODs (edge length: $\sim 99 \mathrm{~nm}$ ). AC feedback control for determining substrate contact and probe positing via hopping mode ${ }^{10-11}$ (Approach-Retract Scanning mode) ${ }^{12}$ was used for SECCM Voltammetric readings were recorded at each pixel in the image, to collect electrochemical images as shown. Hopping mode was found to be superior in preventing probe damage during scanning. ${ }^{10-12}$ All functions and parameters related with SECCM operation was carried by using a commercial software (XEP, Park Systems, South Korea).

A second major issue for utilizing SECCM was positioning of the probe to where nanoparticles are presents in XY dimension of the surface of WE (glassy carbon). Without a certain guide of alignment system, accurate and efficient positioning of the probe and nanometer scale particles is not possible, and also due to surface energy, nanoparticles readily form multi-particle clusters during sample preparation. 

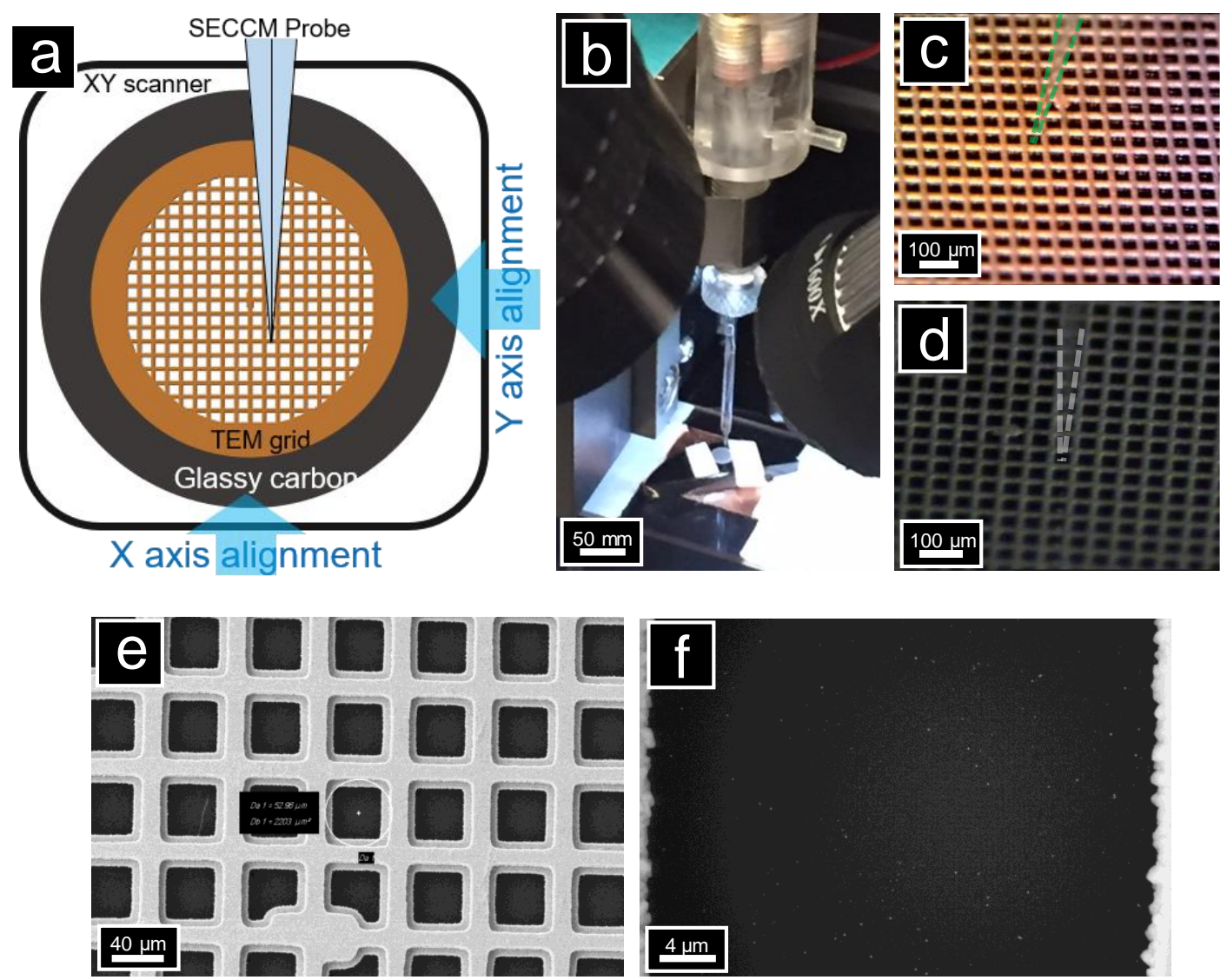

Figure S18. Optically guided probe - nanoparticle alignment system for SECCM. (a) Schematic of the alignment principle for positioning tip of SECCM probe to a predefined square on the TEM grid. The TEM grid was attached to the substrate after nanoparticles were drop-casted. (b) The setup of optically guided probe-nanoparticle alignment system showed a long working distance for the optical microscope (left) and the high-resolution digital microscope (right), projected toward the tip of SECCM probe and the TEM grid with perpendicular orientation relative to each other. (c) An optical micrograph of the long working distance optical microscope for positioning the tip of the SECCM probe (marked by the green dashed line for the $\mathrm{Y}$-axis and (d) an optical micrograph of the high resolution digital microscope as positioning tip of the SECCM probe (marked by the gray dashed line) for the X-axis. (e) Electron micrograph of the TEM grid on a sample and (f) a higher magnification electron micrograph to distinguish single Au nanoparticles. 
system was equipped with two optical microscope having magnification up to $\sim 4000 \mathrm{x}$. After Au nanoparticles were drop-casted onto GC, a TEM grid (3HGC400, Copper, 400 mesh, 3.0 mm hole width $38 \mu \mathrm{m}$ squared, TED PELLA, inc. USA) was taped on top of the GC surface; the SECCM probe was lowered into one of the TEM grid holes (Figure S18). The SECCM probe position relative to the TEM grid was carefully monitored with two optical microscopes positioned $90^{\circ}$ from each other. The probe-tip was carefully lowered into one of the square holes on the TEM grid where single nanoparticle density is about $>24$ particles $/ 100 \mu \mathrm{m}^{2}$ (the squared hole was preidentified with scanning electron microscope, SEM, Auriga 60, Zeiss, Germany). To minimize electron beam effect, the pre-identification step was carried out as low resolution as being able to distinguish single particle against multi-particle cluster (magnification about 10,000 x or less). This SECCM pre-position identification system is highly beneficial for statistical analysis purpose due to the high efficiency to collect a large number of $\mathrm{CVs}$ from only single nanoparticles and to distinguish $\mathrm{Au} \mathrm{NC}$ and $\mathrm{Au}$ OD in mixture sample measurement. 


\section{Section S5. SECCM Cyclic Voltammetric Mapping for HER}

\subsection{Treatment of Extracted CV Data from CV Maps}
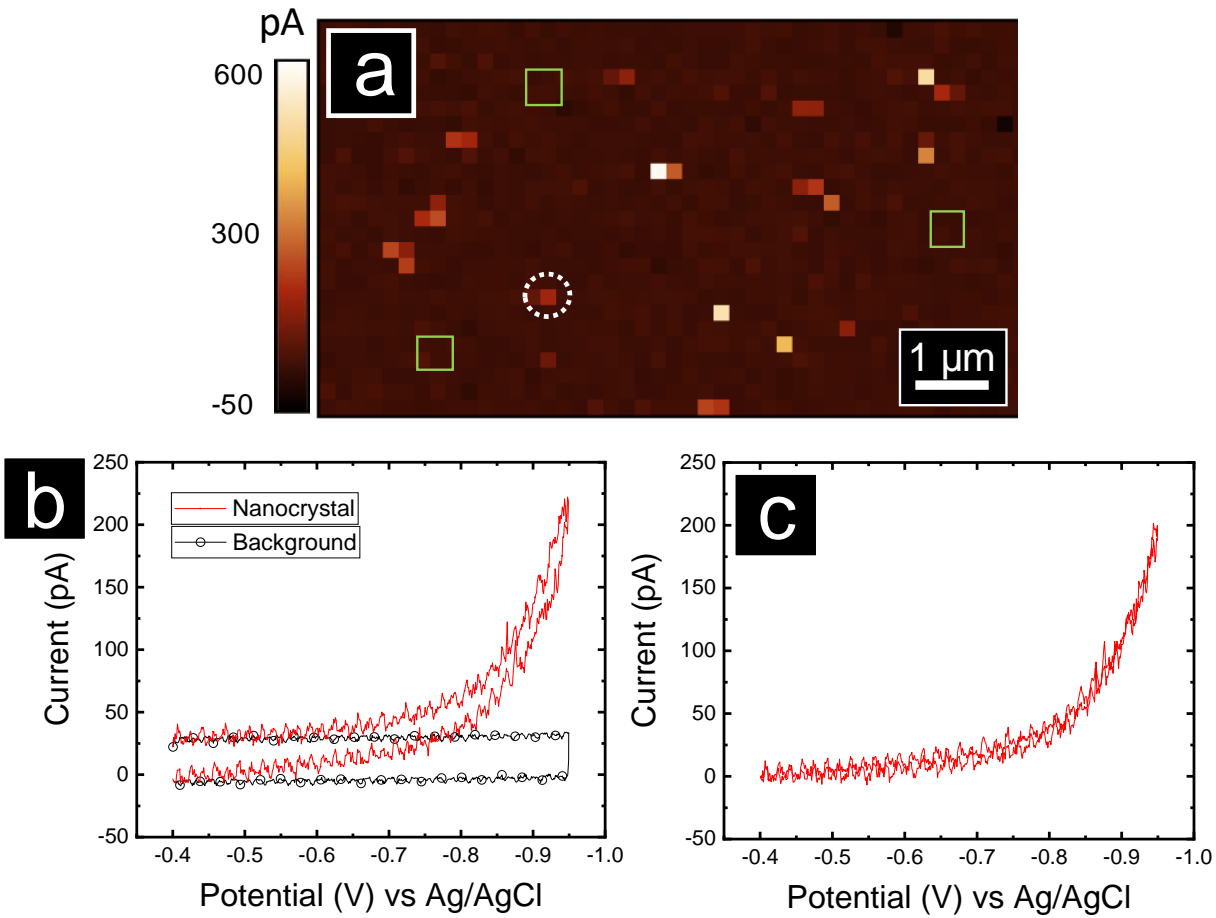

Figure S19. (a) Au cube SECCM CV map at $-0.95 \mathrm{~V}$ vs $\mathrm{Ag} / \mathrm{AgCl}$ where the green squares mark the 3 different areas that were used to determine the averaged CV response for glassy carbon. (b) Raw CV of averaged response of glassy carbon (GC) and a pixel corresponding to a single Au cube (highlighted by the white dotted circle in (a)). (c) CV for a single Au cube after background subtraction (raw $\mathrm{CV}$ for $\mathrm{Au} \mathrm{NC}$ - raw $\mathrm{CV}$ for $\mathrm{GC}$ ). The pixel resolution was $200 \mathrm{~nm}$ in the $\mathrm{X}$ and $\mathrm{Y}$ direction. The solution in the pipette was aqueous $100 \mathrm{mM} \mathrm{HClO}_{4}$ and the scan rate was $1 \mathrm{~V} / \mathrm{s}$. 


\subsection{Maps of Au Cubes}

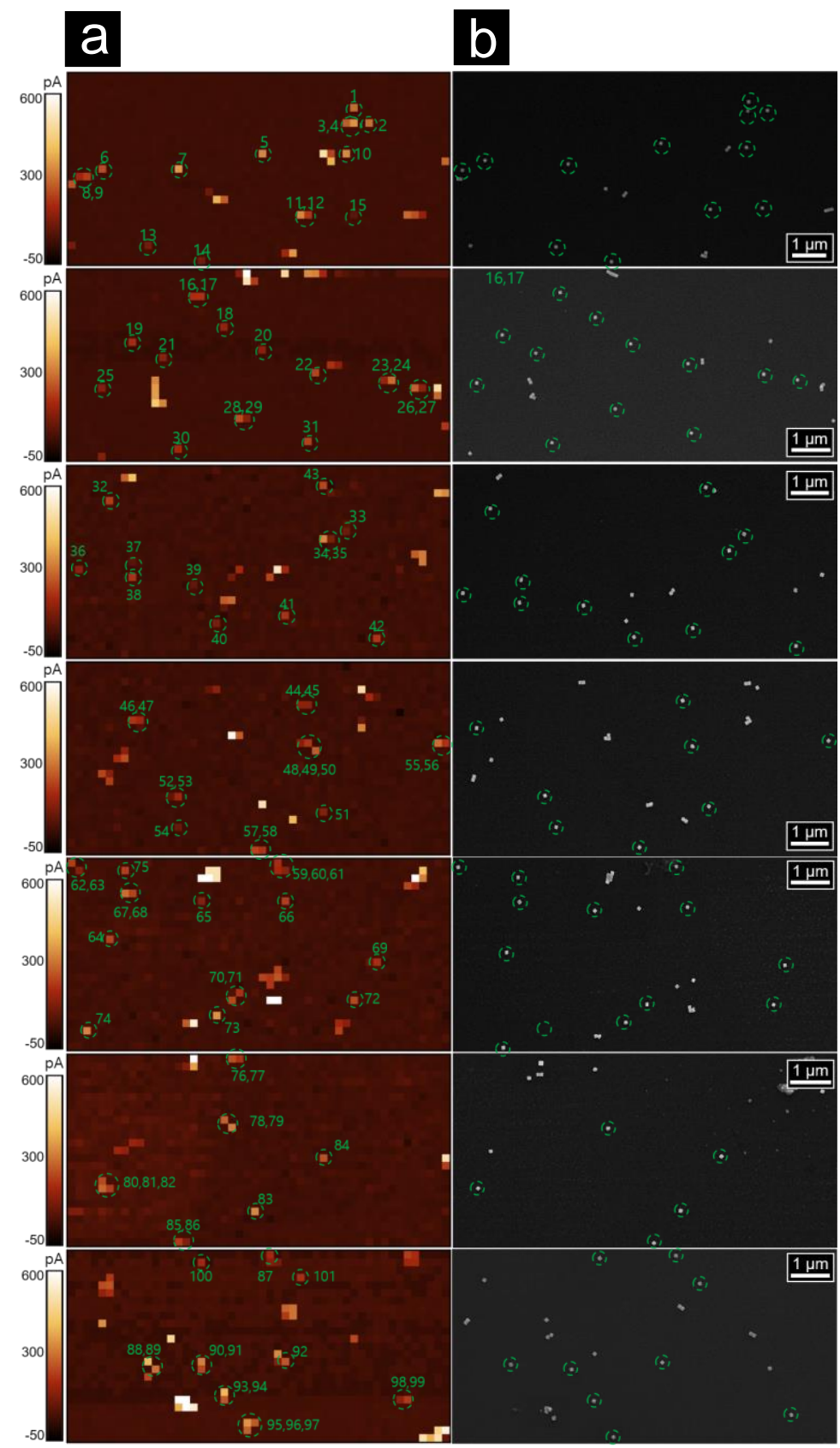

Figure S20. (a) Single Au cubes SECCM CV maps at $-0.95 \mathrm{~V}$ vs $\mathrm{Ag} / \mathrm{AgCl}$ in seven different positions and (b) the corresponding correlative electron micrographs. Total 101 individual CVs of HER that was occurred only at the position of 70 individual single Au cubes (the green dotted circles in electron micrographs highlight the Au cubes that showed activity for HER) were collected for statistical analysis. The solution in the pipette was aqueous $100 \mathrm{mM} \mathrm{HClO}_{4}$ and the scan rate was $1 \mathrm{~V} / \mathrm{s}$. Pixel resolution is $250 \mathrm{~nm}$ for both $\mathrm{X}$ and $\mathrm{Y}$ axis with scan size $10 \mu \mathrm{m}$ $\mathrm{x} 5 \mu \mathrm{m}$. 


\subsection{Maps of Au Octahedra}

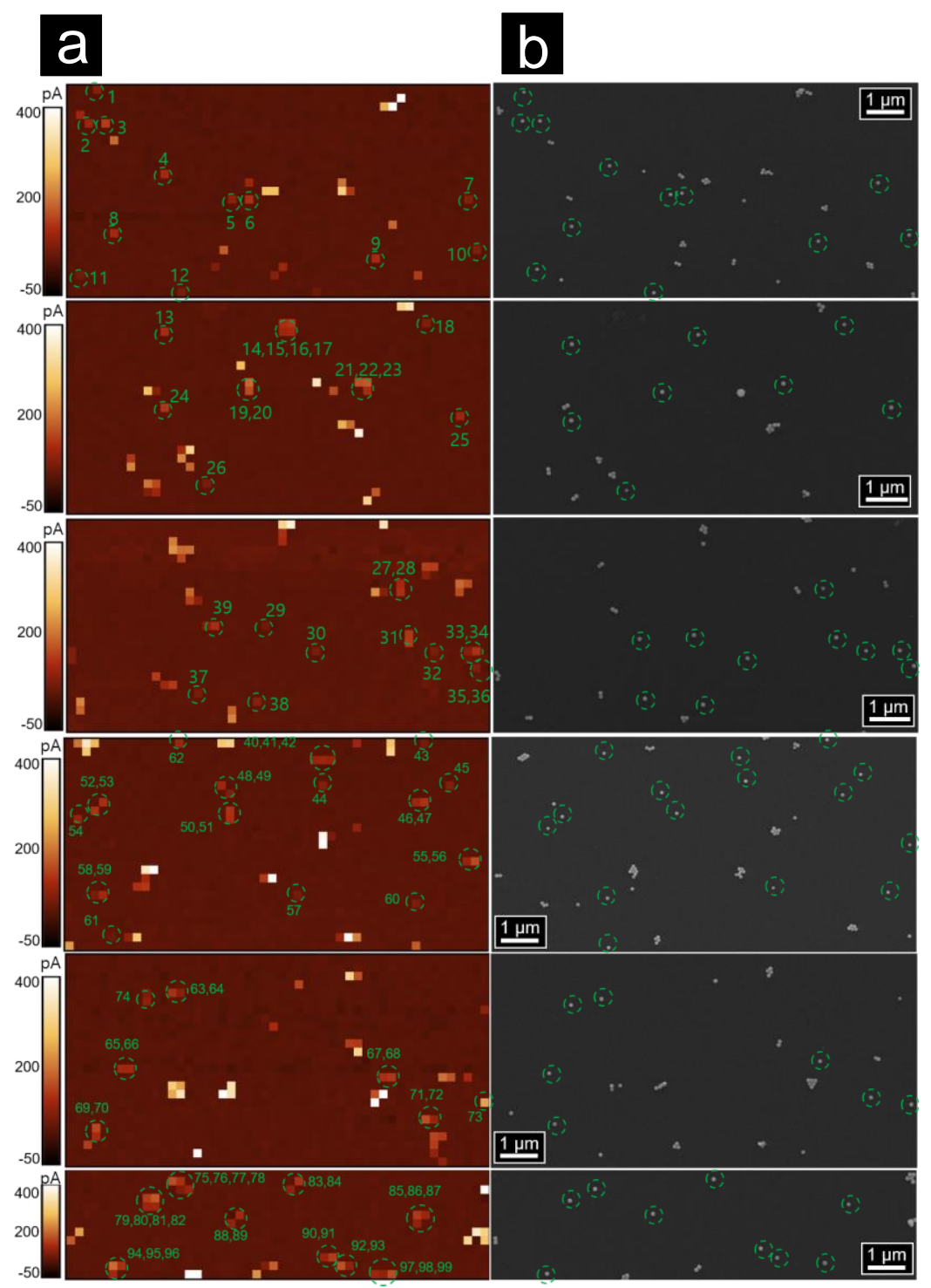

Figure S21. (a)Au octahedra SECCM CV map collected at $-1.05 \mathrm{~V}$ vs $\mathrm{Ag} / \mathrm{AgCl}$ for the HER and (b) the corresponding correlative electron micrographs for three different positions (66 single AuODs for 99 pixel data). The green dotted circles in electron micrographs highlight the single $\mathrm{Au}$ octahedra that showed activity for HER/ORR were collected for statistical analysis. The pixel resolution was $200 \mathrm{~nm}$ in the $\mathrm{X}$ and $\mathrm{Y}$ direction with a scan size of $10 \mu \mathrm{m}$ by $5 \mu \mathrm{m}$. The solution in the pipette was aqueous $100 \mathrm{mM} \mathrm{HClO}_{4}$ and the scan rate was $1 \mathrm{~V} / \mathrm{s}$. 


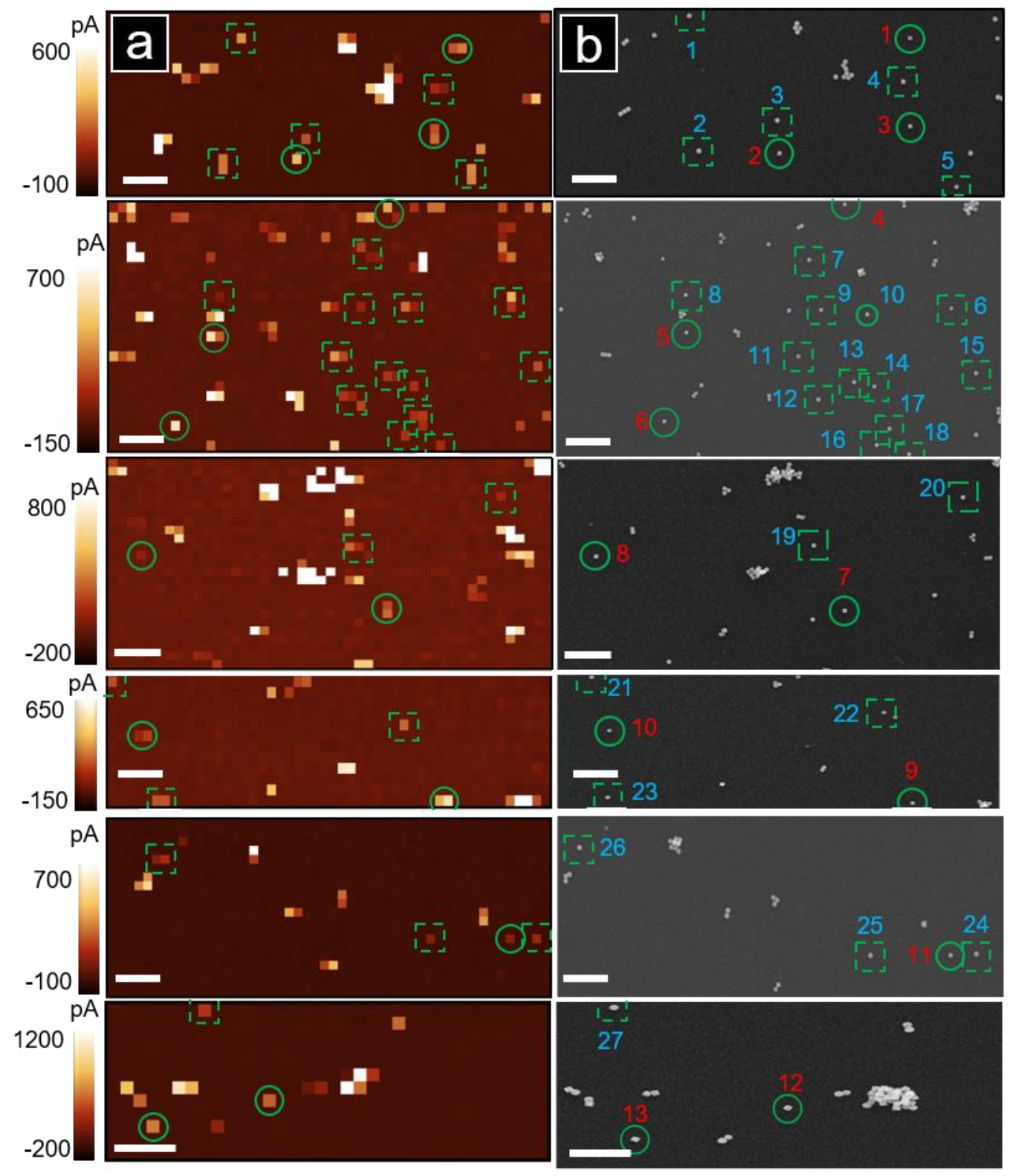

Figure S22. (a) SECCM CV maps at $-1.05 \mathrm{~V}$ vs $\mathrm{Ag} / \mathrm{AgCl}$ for a mixture of $\mathrm{Au} \mathrm{NCs}$ and $\mathrm{Au}$ ODs at six different positions and (b) the corresponding correlative electron micrographs. The solid green circles and dashed green squares highlight the single $\mathrm{Au}$ NCs and Au ODs, respectively. The scale bars in (a) and (b) are $1 \mu \mathrm{m}$. In total, 13 and 27 isolated Au NCs and $\mathrm{Au}$ ODs which correspond to 20 and 43 pixels, respectively were measured. The solution in the pipette was aqueous $100 \mathrm{mM} \mathrm{HClO}_{4}$ and the scan rate was $1 \mathrm{~V} / \mathrm{s}$. Pixel resolution is 200 $\mathrm{nm}$ for both $\mathrm{X}$ and $\mathrm{Y}$ axis. 


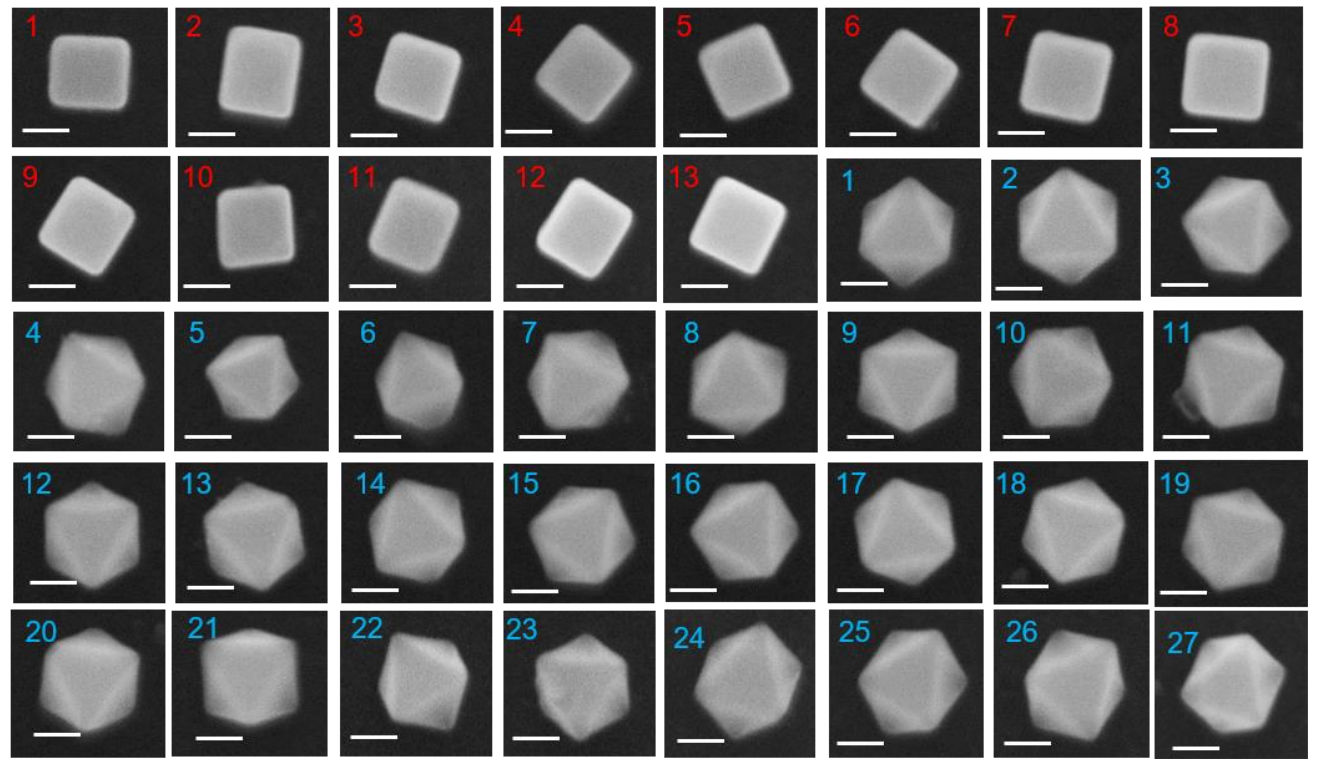

Figure S23. High-resolution electron micrographs of Au NCs (red numbers) and Au ODs (blue numbers) that correspond to the CV maps and correlative electron micrographs in Figure S22.

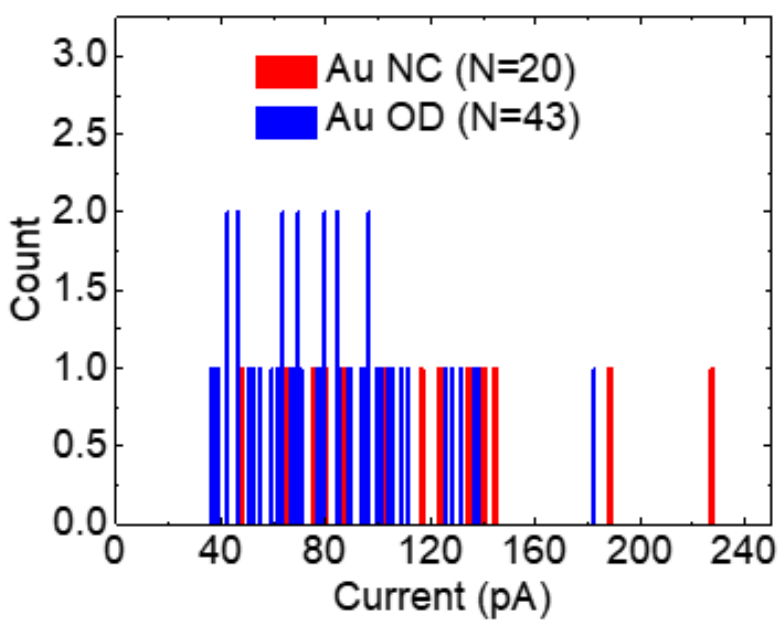

Figure S24. Histogram of current magnitude at $-0.95 \mathrm{~V}$ vs $\mathrm{Ag} / \mathrm{AgCl}$ when a mixture of $\mathrm{Au}$ NCs and ODs on the same sample were studied by SECCM CV mapping. A total of 13 individual $\mathrm{Au} \mathrm{NCs}$ and $27 \mathrm{Au} \mathrm{ODs}$, corresponding to 20 and 43 pixels, respectively, were studied. 

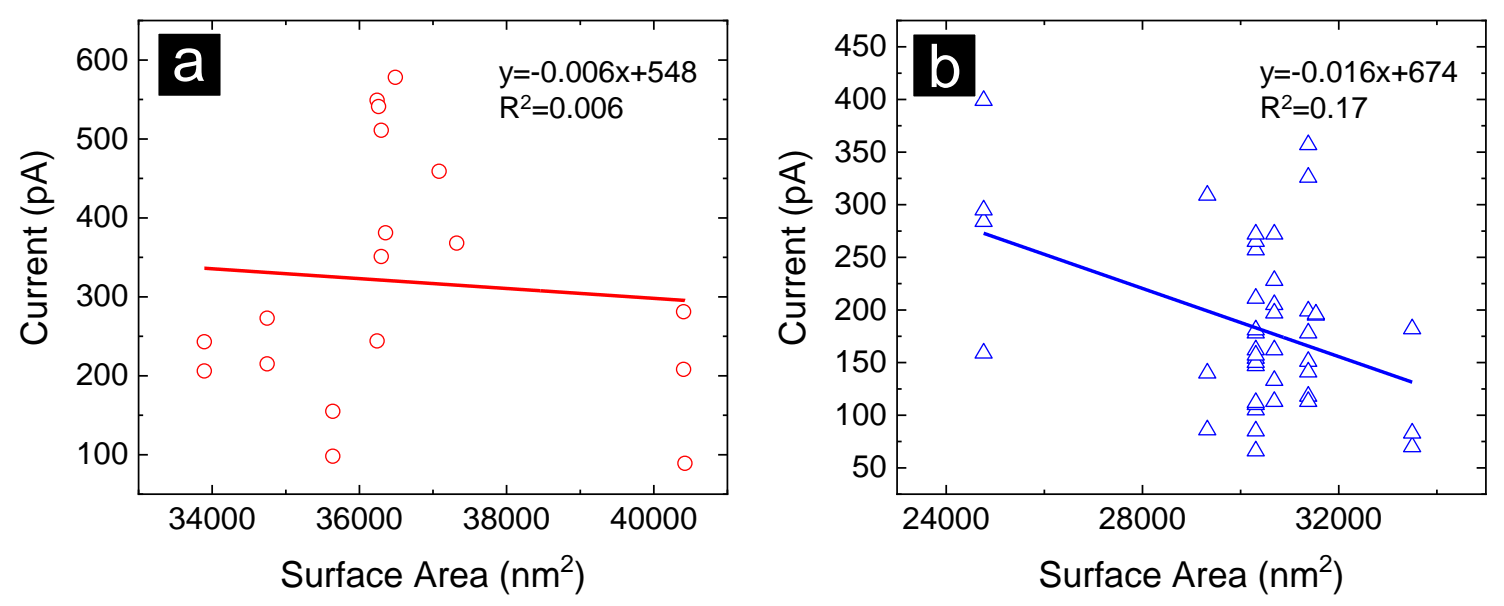

Figure S25. Relationship between surface area of individual (a) Au NCs and (b) ODs in the mixture sample (Figures S20-21) and current magnitude at $-1.05 \mathrm{~V}$ vs $\mathrm{Ag} / \mathrm{AgCl}$. All pixels that correspond to single Au NCs and ODs are included. 


\section{Section S6. Finite Element Method (FEM) Simulations and}

\section{Experimental Result Comparison of SECCM CV Measured at}

\section{Individual Particles}

6.1 Voltammetric Response of NC and OD Particles as Function of NP Position within The

Droplet
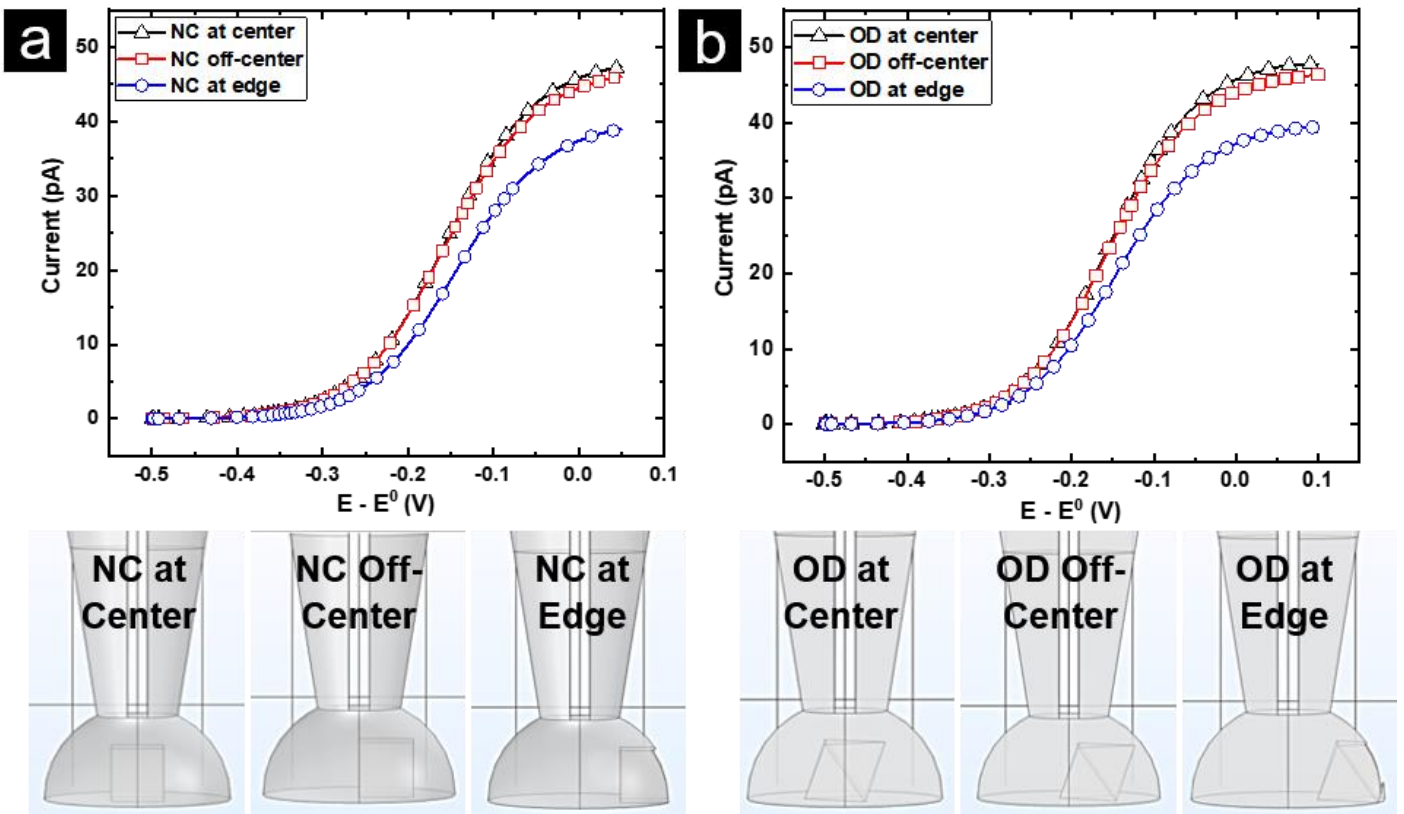

Figure S26. Simulated cyclic voltammograms for ferrocyanide oxidation at a NC (a) and OD

(b) when the particle is at the center, off-center, and edge of the SECCM droplet cell. A

schematic showing the position of the particle in the droplet is shown below the cyclic voltammograms. 


\section{Concentrations}

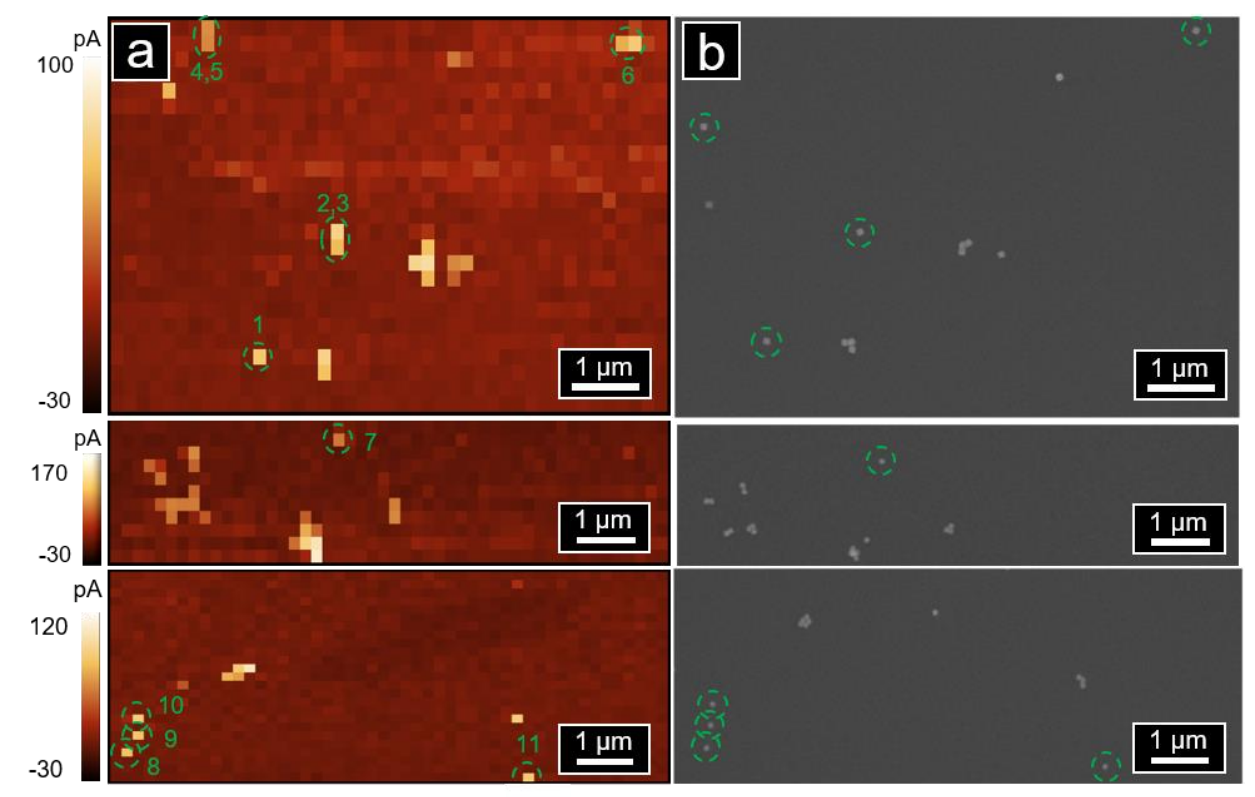

Figure S27. SECCM CV maps at $-1.35 \mathrm{~V}$ vs $\mathrm{Ag} / \mathrm{AgCl}$ at three different positions and (b) the corresponding correlative electron micrographs. The scale bar is $1 \mu \mathrm{m}$. The solution in the pipette was aqueous $2 \mathrm{mM} \mathrm{HClO}_{4}$ and $10 \mathrm{mM} \mathrm{KClO}_{4}$ and the scan rate was $1 \mathrm{~V} / \mathrm{s}$. Pixel resolution is $250 \mathrm{~nm}$ for both $\mathrm{X}$ and $\mathrm{Y}$ axis. 

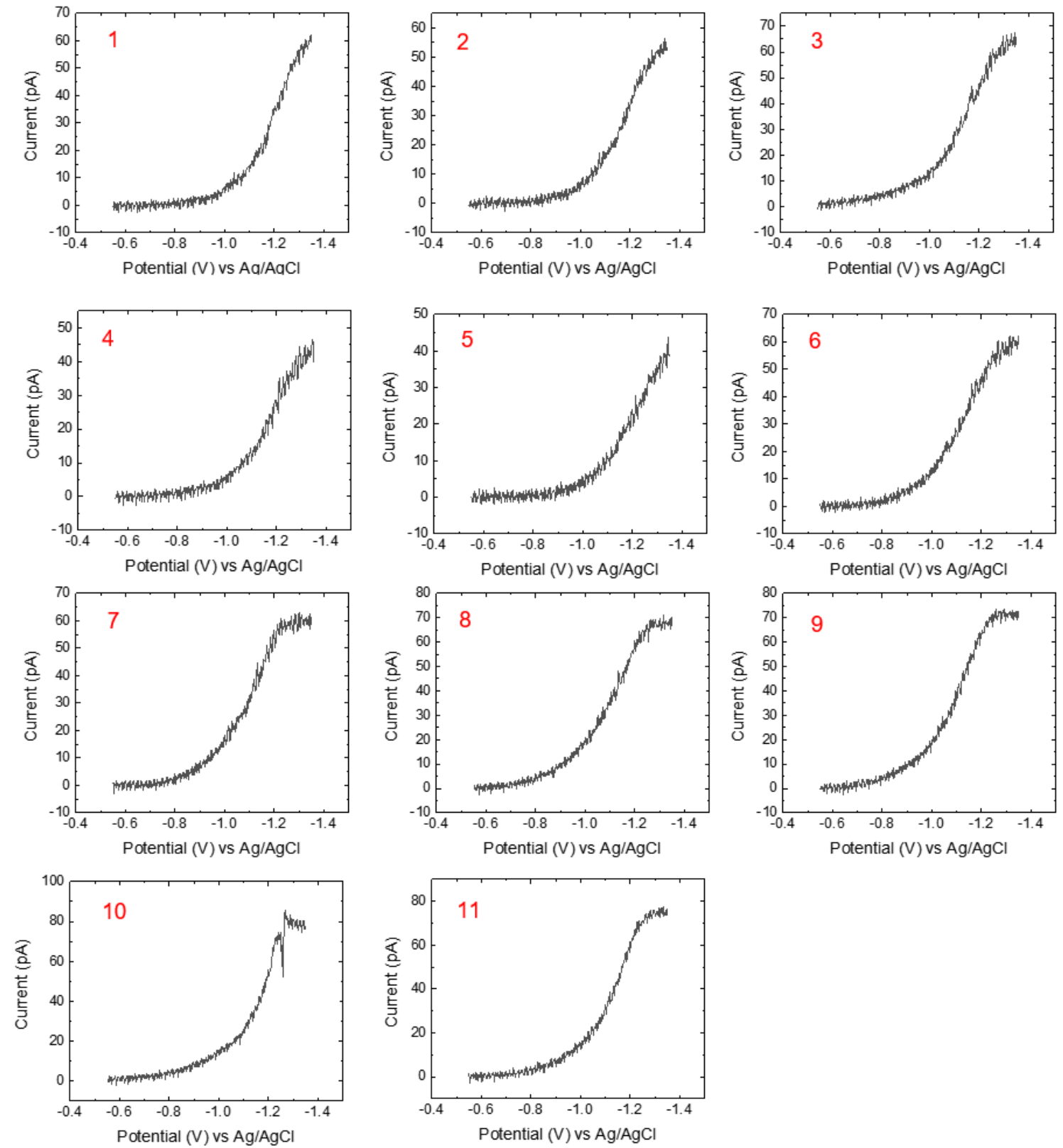

Figure S28. Extracted CVs for single Au NCs that correspond to the red numbers in figure S27. The solution in the pipette was aqueous $2 \mathrm{mM} \mathrm{HClO}_{4}$ and $10 \mathrm{mM} \mathrm{KClO}_{4}$ and the scan rate was $1 \mathrm{~V} / \mathrm{s}$. 
Finite element simulation of HER on NC inside the SECCM drop was also carried out to compare the simulated results with experimentally obtained CVs at single NCs. The same SECCM pipette probe and drop model was used as described in Section 6.1. To simulate the HER reaction, with a starting electrolyte concentration of $2 \mathrm{mM}$ perchloric acid in $10 \mathrm{mM} \mathrm{KCl}$, the following parameters were utilized: diffusion coefficients $\mathrm{H}^{+}-9.3 \times 10^{-5} \mathrm{~cm}^{2} / \mathrm{s}, \mathrm{H}_{2}-3.7 \times 10^{-5} \mathrm{~cm}^{2} / \mathrm{s}, \mathrm{K}^{+}$$1.957 \times 10^{-9} \mathrm{~m}^{2} / \mathrm{s}, \mathrm{ClO}_{4}{ }^{-}-1.792 \times 10^{-9} \mathrm{~m}^{2} / \mathrm{s},{ }^{15}$ reaction rate $-0.045 \mathrm{~cm} / \mathrm{s}^{16}$ and scan rate $-1 \mathrm{~V} / \mathrm{s}$. The simulated CV with experimental results is shown in Figure S29.
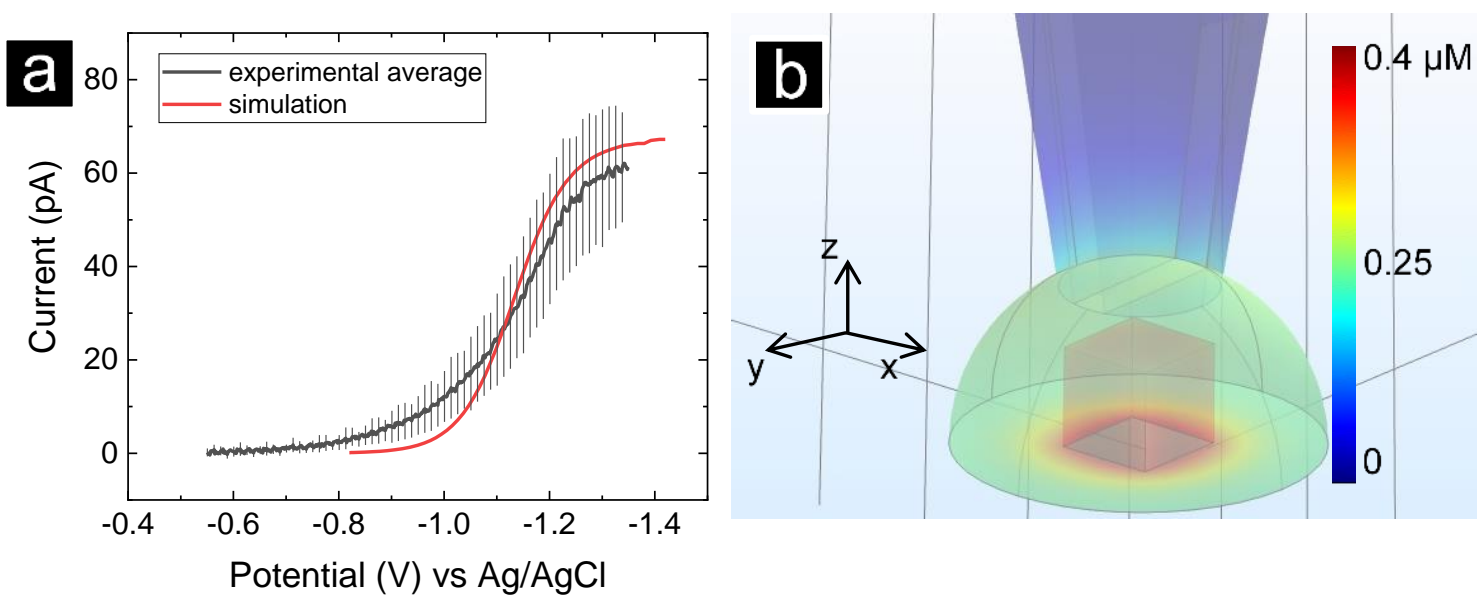

Figure S29. (a) Experimental average ( $\mathrm{N}=11)$ (black) and simulated (red) voltammograms for HER at an individual Au NC. (b) Simulated product concentration profile at a single Au NC at $\mathrm{t}=0$ when the droplet diameter and height are $280 \mathrm{~nm}$ and $125 \mathrm{~nm}$, respectively, and the pipette tip radius is $60 \mathrm{~nm}$. The solution in the pipette was aqueous $2 \mathrm{mM} \mathrm{HClO}_{4}$ in $10 \mathrm{mM} \mathrm{KClO}_{4}$. The scan rate was $1 \mathrm{~V} / \mathrm{s}$. 


\subsection{Study of Geometric Effect on Electrochemical Response Between NC and OD}

To elucidate the effect of nanoparticle shape on the electrochemical response, 3D FEM simulations on cube $\left(\mathrm{NC}\right.$, edge length $=78 \mathrm{~nm}$, surface area of five faces $\left.\sim 30420 \mathrm{~nm}^{2}\right)$ and octahedra (OD, edge length $=99 \mathrm{~nm}$, surface area of seven faces $\sim 29707 \mathrm{~nm}^{2}$ ) nanoparticles in SECCM probe setup were carried out. A report file for an NC is attached in Section 6.4 for reference. The geometric parameters of the theta pipette probe, tip radius $\left(r_{p}\right)=60 \mathrm{~nm}$ and cone angle $=9^{\circ}$, were obtained from STEM images of pipettes used in the SECCM experiments. An estimated drop height (htd) of $125 \mathrm{~nm}$ and a hemisphere shape were implemented for the drop formed between the pipette and the surface in SECCM. A potential difference of $100 \mathrm{mV}$ was set between the two barrels of the pipette probe. Butler-Volmer kinetics were applied on the nanoparticle surfaces for ferrocyanide oxidation to ferricyanide reaction. Starting concentration of $7.8 \mathrm{mM}$ potassium ferrocyanide in $10 \mathrm{mM} \mathrm{KCl}$ and zero concentration of ferricyanide ions were implemented. Following parameters were used to simulated the electrochemical reaction: diffusion coefficients of $\mathrm{K}^{+}-1.957 \times 10^{-9} \mathrm{~m}^{2} / \mathrm{s}, \mathrm{Cl}^{-}-2.032 \times 10^{-9} \mathrm{~m}^{2} / \mathrm{s},{ }^{17}\left[\mathrm{Fe}(\mathrm{CN})_{6}\right]^{4-}-6.1 \times 10^{-6} \mathrm{~cm}^{2} / \mathrm{s}$, $\left[\mathrm{Fe}(\mathrm{CN})_{6}\right]^{3-}-5.5 \times 10^{-6} \mathrm{~cm}^{2} / \mathrm{s}$, reaction rate $\left(\mathrm{k}_{0}\right), 0.01 \mathrm{~cm} / \mathrm{s}^{18}$ and $-0.36 \mathrm{~V}$ formal potential $\left(\mathrm{E}^{0}\right) .{ }^{19}$ The cyclic voltammograms for each model were simulated at a scan rate of $1 \mathrm{~V} / \mathrm{s}$. Figure $\mathbf{S 3 0}$ shows the resulting cyclic voltammograms from a single NC (red) and a single OD (blue). The two voltammograms overlap very well over each other, indicating that when the kinetic conditions are same, the shape of the nanoparticle at the center of the SECCM drop does not affect mass transport during an electrochemical reaction. 


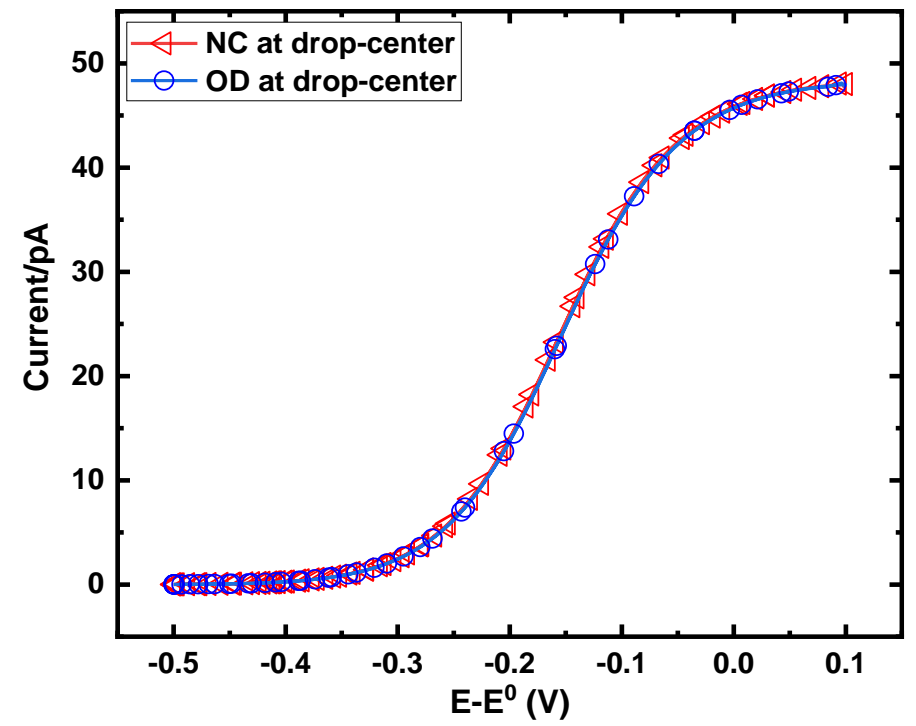

Figure S30. Plot of simulated cyclic voltammograms for ferrocyanide oxidation on NC (red) and OD (blue) at the center of the drop formed in the tip of SECCM probe. 


\subsection{FEM Simulations Report File}

Global settings

Name SECCM np cube center CV-improv 1 10-2-19.mph

Version COMSOL Multiphysics 5.4 (Build: 246)

Used products

COMSOL Multiphysics

Chemical Reaction Engineering Module

Electrochemistry Module

Parameters

Parameters 1

\begin{tabular}{|c|c|c|c|}
\hline Name & Expression & Value & Description \\
\hline $\mathrm{e}$ & $78[\mathrm{~nm}]$ & $7.8 \mathrm{E}-8 \mathrm{~m}$ & Edge length of cubic np \\
\hline $\mathrm{v}$ & $1[\mathrm{~V} / \mathrm{s}]$ & $1 \mathrm{~V} / \mathrm{s}$ & Voltammetry scan rate \\
\hline c_bulk & $7.8[\mathrm{mmol} / \mathrm{L}]$ & $7.8 \mathrm{~mol} / \mathrm{m}^{3}$ & Reactant bulk concentration \\
\hline D1 & $6.1 \mathrm{e}-6\left[\mathrm{~cm}^{2} / \mathrm{s}\right]$ & $6.1 \mathrm{E}-10 \mathrm{~m}^{2} / \mathrm{s}$ & $\begin{array}{l}\text { Reactant diffusion } \\
\text { coefficient }\end{array}$ \\
\hline D2 & $5.5 \mathrm{e}-6\left[\mathrm{~cm}^{2} / \mathrm{s}\right]$ & $5.5 \mathrm{E}-10 \mathrm{~m}^{2} / \mathrm{s}$ & Product diffusion coefficient \\
\hline k0 & $0.01[\mathrm{~cm} / \mathrm{s}]$ & $1 \mathrm{E}-4 \mathrm{~m} / \mathrm{s}$ & Reaction rate \\
\hline Cdl & $0.2\left[\mathrm{~F} / \mathrm{m}^{2}\right]$ & $0.2 \mathrm{~F} / \mathrm{m}^{2}$ & Double layer capacitance \\
\hline $\mathrm{T}$ & $298.15[\mathrm{~K}]$ & $298.15 \mathrm{~K}$ & Temperature \\
\hline
\end{tabular}




\begin{tabular}{|l|l|l|l|}
\hline Name & Expression & Value & Description \\
\hline E_start & $-0.5[\mathrm{~V}]$ & $-0.5 \mathrm{~V}$ & Start potential \\
\hline E_vertex & $0.1[\mathrm{~V}]$ & $0.1 \mathrm{~V}$ & Switching potential \\
\hline Ef & $-0.36[\mathrm{~V}]$ & $-0.36 \mathrm{~V}$ & Formal potential \\
\hline rdrop & sqrt $\left(\left(\mathrm{rp}^{2}\right)+\left(\mathrm{htd}^{2}\right)\right)$ & $1.3865 \mathrm{E}-7 \mathrm{~m}$ & Radius of drop \\
\hline htd & $125[\mathrm{~nm}]$ & $1.25 \mathrm{E}-7 \mathrm{~m}$ & Height of drop \\
\hline theta & $9[\mathrm{deg}]$ & $0.15708 \mathrm{rad}$ & Pipette cone angle \\
\hline D3 & $1.957 \mathrm{E}-9\left[\mathrm{~m}^{2} / \mathrm{s}\right]$ & $1.957 \mathrm{E}-9 \mathrm{~m} / \mathrm{s}$ & Diffusion coefficient of K+ \\
\hline c_elc & $10[\mathrm{mM}]$ & $10 \mathrm{~mol} / \mathrm{m}^{3}$ & Concentration of electrolyte \\
\hline rp & $60[\mathrm{~nm}]$ & $6 \mathrm{E}-8 \mathrm{~m}$ & Pipette tip radius \\
\hline D4 & $2.032 \mathrm{E}-9\left[\mathrm{~m}^{2} / \mathrm{s}\right]$ & $2.032 \mathrm{E}-9 \mathrm{~m}^{2} / \mathrm{s}$ & Diffusion coefficient of Cl- \\
\hline
\end{tabular}

\section{Component 1}

Definitions

Coordinate Systems

Boundary System 1

\begin{tabular}{|l|l|}
\hline Coordinate system type & Boundary system \\
\hline Tag & sys1 \\
\hline
\end{tabular}

Coordinate names

\begin{tabular}{|l|l|l|}
\hline First & Second & Third \\
\hline t1 & t2 & $\mathrm{n}$ \\
\hline
\end{tabular}




\section{Geometry 1}

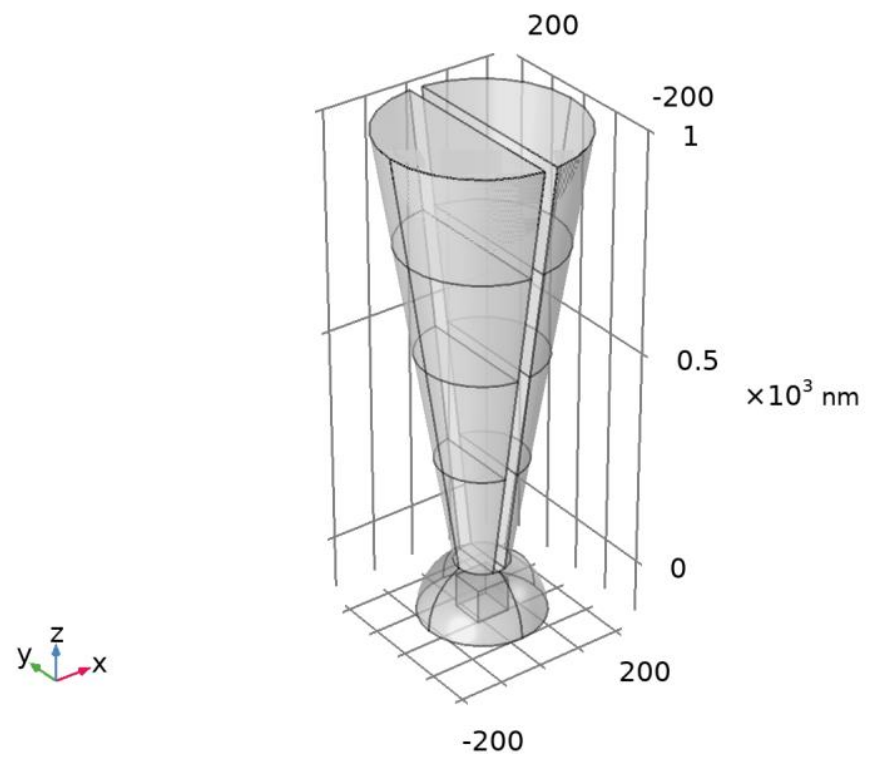

Geometry 1

Units

\begin{tabular}{|l|l|}
\hline Length unit & $\mathrm{nm}$ \\
\hline Angular unit & $\mathrm{deg}$ \\
\hline
\end{tabular}

Materials

Water 


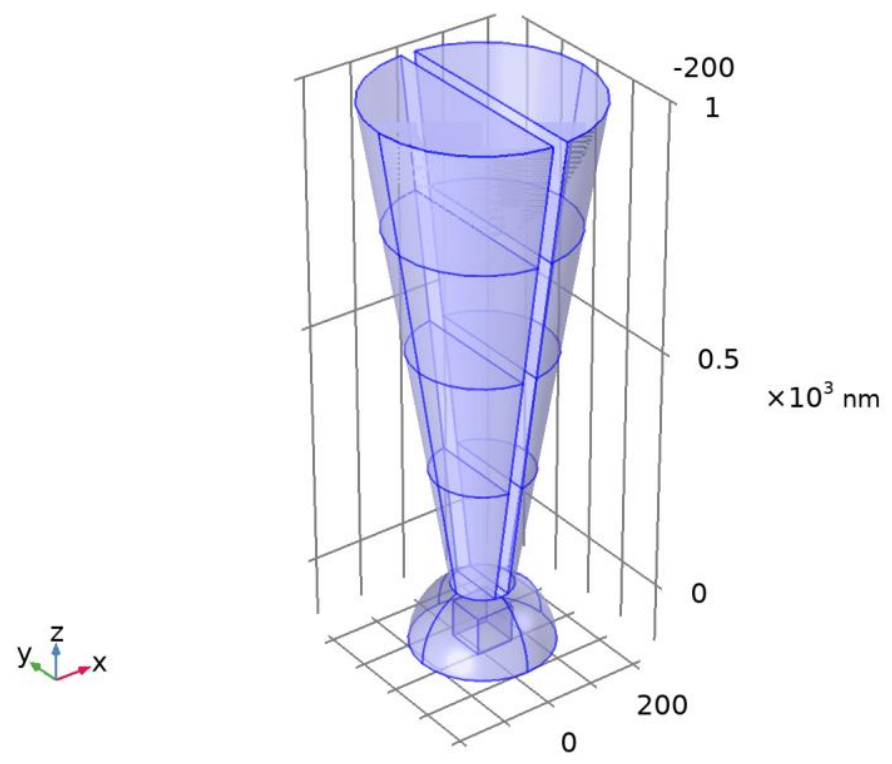

Water

Selection

\begin{tabular}{|l|l|}
\hline Geometric entity level & Domain \\
\hline Selection & Domains 1-9 \\
\hline
\end{tabular}

Electroanalysis

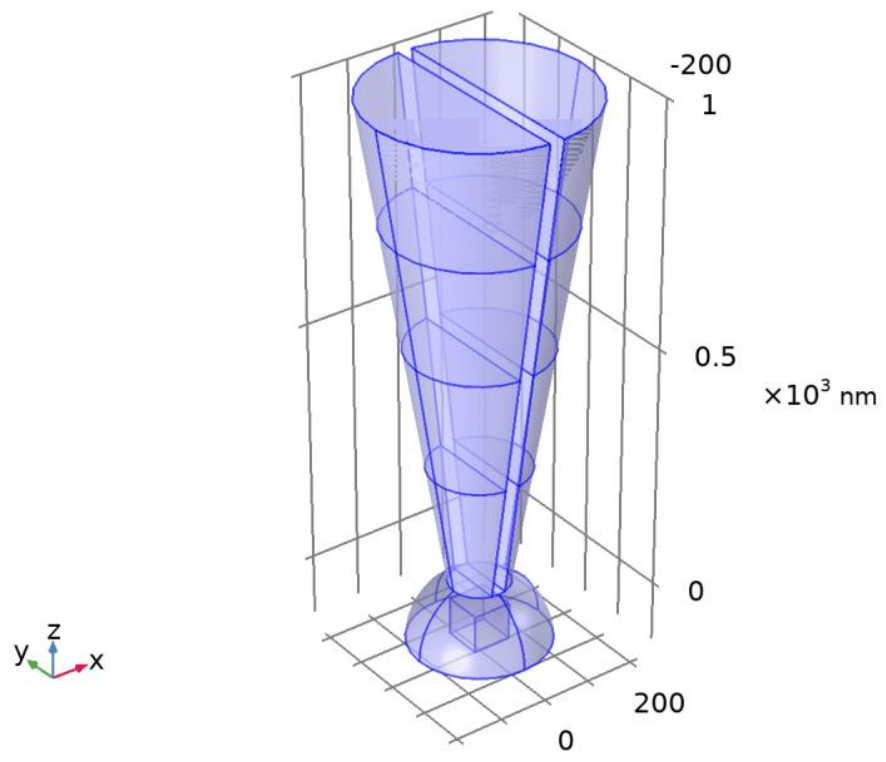

Electroanalysis 
Equations

$\frac{\partial c_{i}}{\partial t}+\nabla \cdot \mathbf{J}_{i}=R_{i}$

$\mathbf{J}_{i}=-D_{i} \nabla c_{i}-z_{i} u_{m, j} F c_{i} \nabla \phi_{1}$

Features

Transport Properties 1

No Flux 1

Initial Values 1

Concentration 1

Electrode Surface 1

Transport Properties 1

Equations

$\frac{\partial c_{i}}{\partial t}+\nabla \cdot \mathbf{J}_{i}=R_{i}$

$\mathbf{J}_{i}=-D_{i} \nabla c_{i}-z_{i} u_{\mathrm{m},} F c_{i} \nabla \phi_{1}$

No Flux 1

Equations

$-\mathbf{n} \cdot \mathbf{J}_{i}=0$

Concentration 1

Equations

$c_{i}=c_{0, j}$

Electrode Surface 1

Equations 
$i_{\text {total }}=\sum_{m} i_{\text {loc, } m}+i_{\mathrm{dl}}$

$-\mathbf{n} \cdot \mathbf{J}_{i}=R_{i, \text { tot }}, \quad R_{i, \text { tot }}=\sum_{. . m \ldots \ldots . . .} R_{i, m}$

Electrode Reaction 1

Equations

$\eta=\phi_{\mathrm{s}, \mathrm{ext}}-\phi_{\mathrm{l}}-E_{\mathrm{eq}}$

Electrostatics

$y+2$

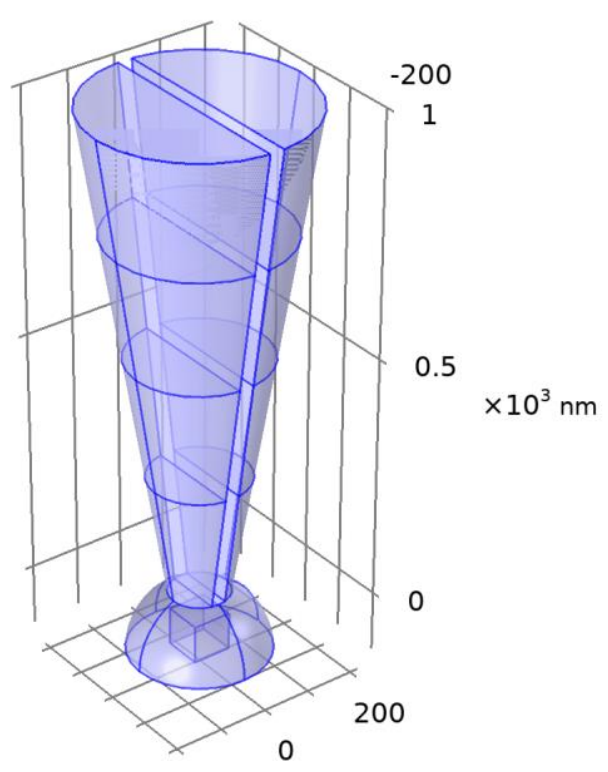

Electrostatics

Equations

$\nabla \cdot \mathbf{D}=\rho_{v}$

$\mathbf{E}=-\nabla V$

Features

Charge Conservation 1

Zero Charge 1 
Initial Values 1

Electric Potential 1

Ground 1

Charge Conservation 1

Equations

$\mathbf{E}=-\nabla V$

$\nabla \cdot\left(\epsilon_{0} \epsilon_{\mathrm{r}} \mathbf{E}\right)=\rho_{\mathrm{v}}$

Zero Charge 1

Equations

$\mathbf{n} \cdot \mathbf{D}=0$

Electric Potential 1

Equations

$V=V_{0}$

Ground 1

Equations

$V=0$.

Multiphysics

Potential Coupling 1 


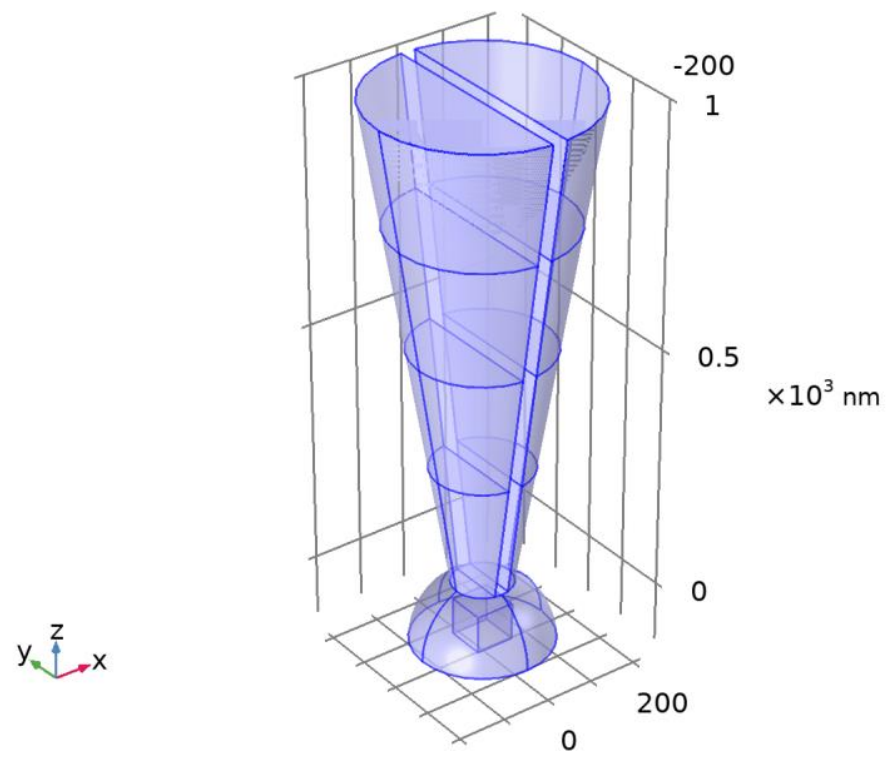

Potential Coupling 1

Mesh 1

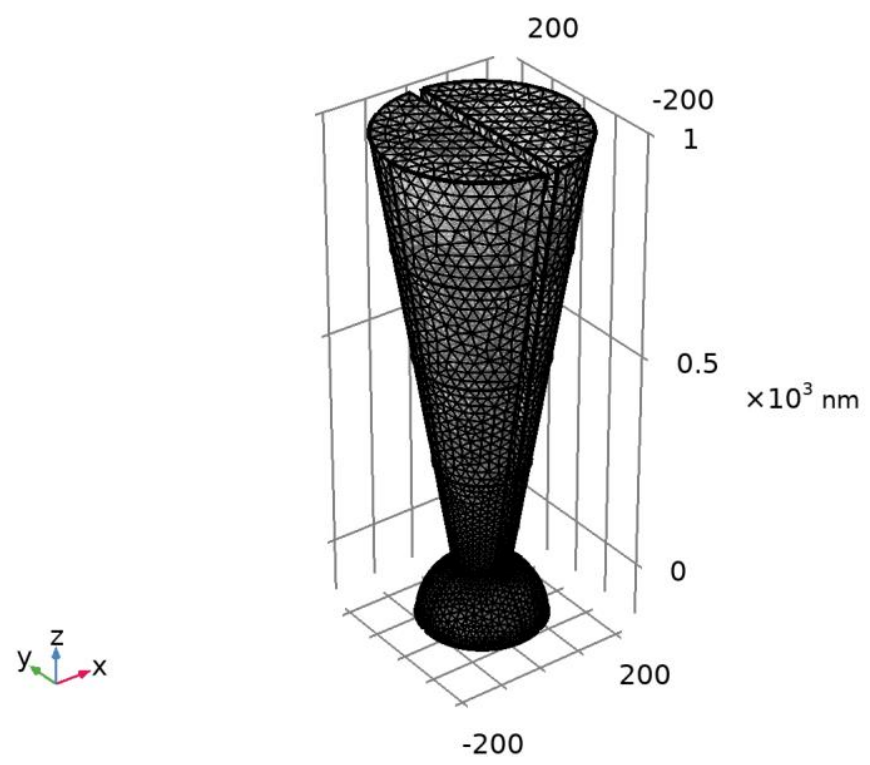

Mesh 1

Study 1

Computation information

Computation time $\quad 1 \mathrm{~h} 28 \mathrm{~min} 42 \mathrm{~s}$ 


\begin{tabular}{|l|l|}
\hline CPU & Intel(R) Xeon(R) CPU E5-2650 v2@2.60GHz, 16 cores \\
\hline Operating system & Linux
\end{tabular}

Cyclic Voltammetry

Study settings

\begin{tabular}{|l|l|}
\hline Description & Value \\
\hline Include geometric nonlinearity & Off \\
\hline
\end{tabular}

Values of dependent variables

\begin{tabular}{|l|l|}
\hline Description & Value \\
\hline Settings & User controlled \\
\hline Method & Solution \\
\hline Study & $\underline{\text { Study } 1}$ \\
\hline
\end{tabular}

Mesh selection

\begin{tabular}{|l|l|}
\hline Geometry & Mesh \\
\hline mesh1 & mesh1 \\
\hline
\end{tabular}

Physics and variables selection

\begin{tabular}{|l|l|}
\hline Physics interface & Discretization \\
\hline Electroanalysis (elan) & physics \\
\hline Electrostatics (es) & physics \\
\hline
\end{tabular}


Mesh selection

\begin{tabular}{|l|l|}
\hline Geometry & Mesh \\
\hline Geometry 1 (geom1) & mesh1 \\
\hline
\end{tabular}

Results

Data Sets

Study $1 /$ Solution 1

Solution

\begin{tabular}{|l|l|}
\hline Description & Value \\
\hline Solution & Solution 1 \\
\hline Component & Save Point Geometry 1 \\
\hline
\end{tabular}

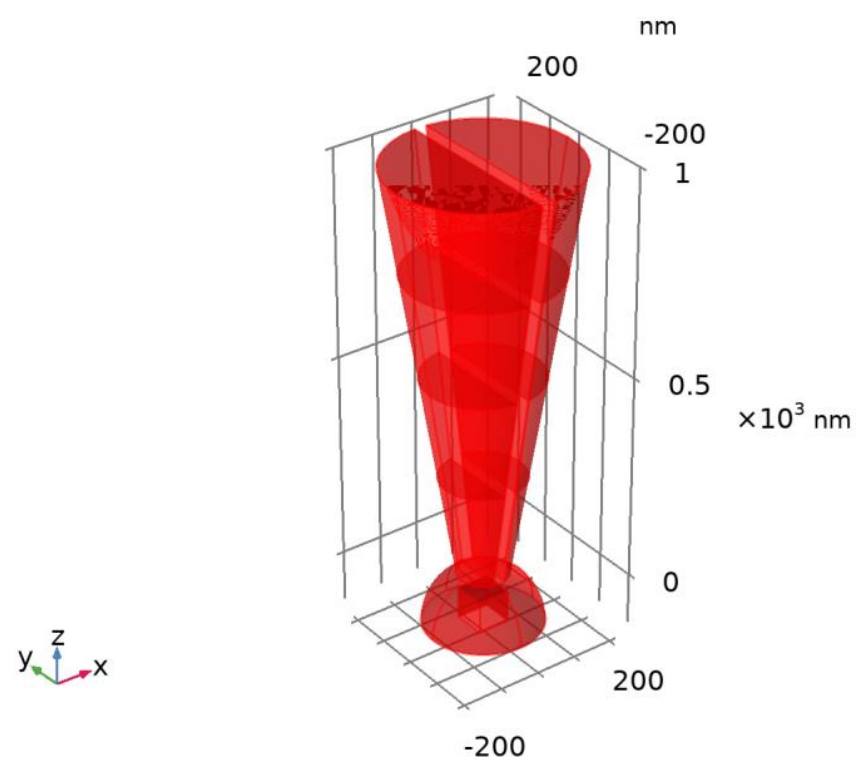

Data set: Study 1/Solution 1

Plot Groups

Cyclic Voltammograms (elan) 


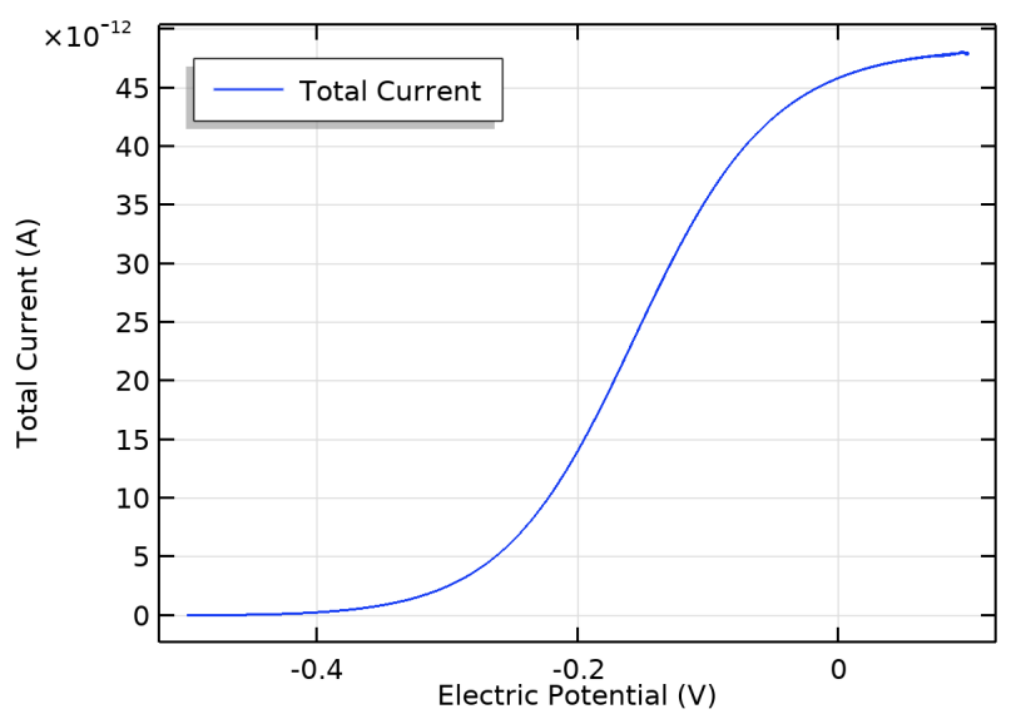

Electrode Potential (elan)

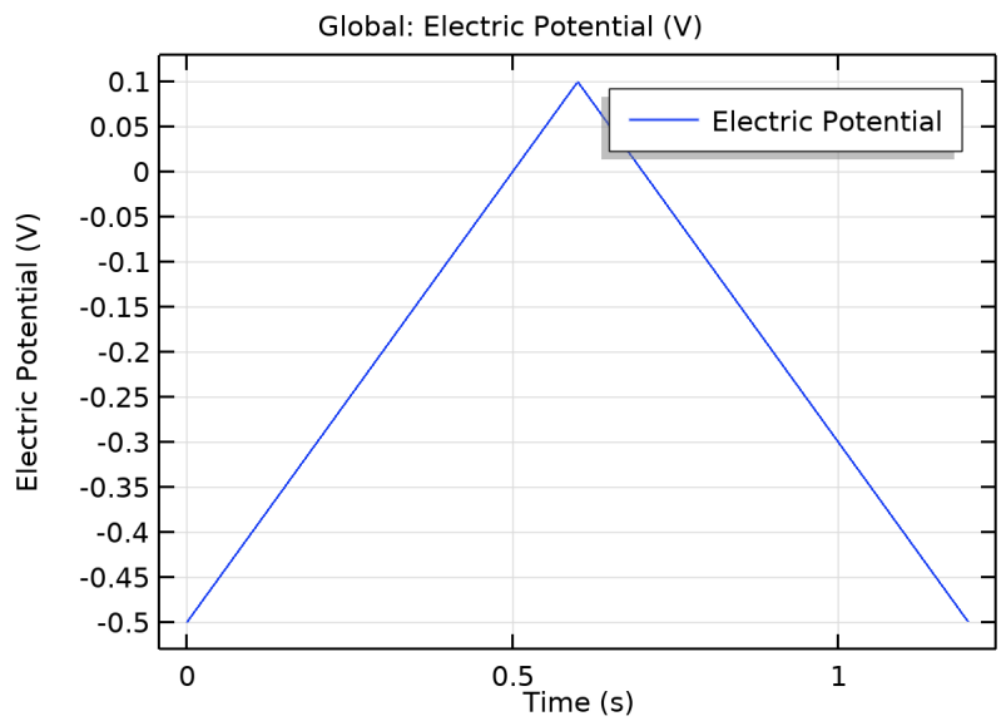

Global: Electric Potential (V)

Average Current Density (elan) 


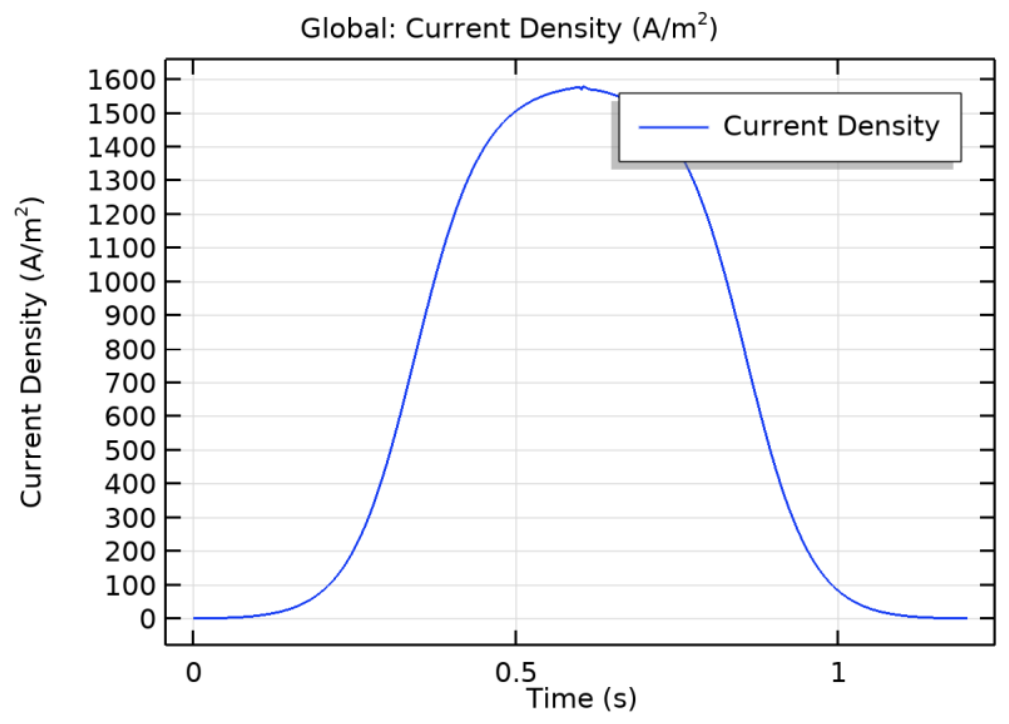

Global: Current Density $\left(\mathrm{A} / \mathrm{m}^{2}\right)$

Concentration (elan)

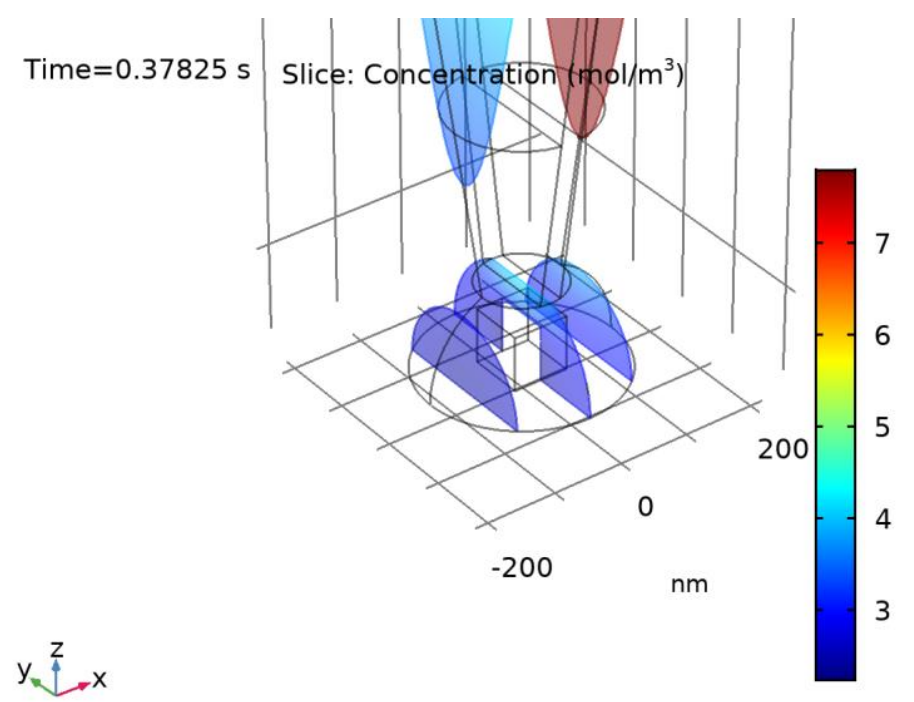

Slice: Concentration $\left(\mathrm{mol} / \mathrm{m}^{3}\right)$

Concentration (elan) 1 


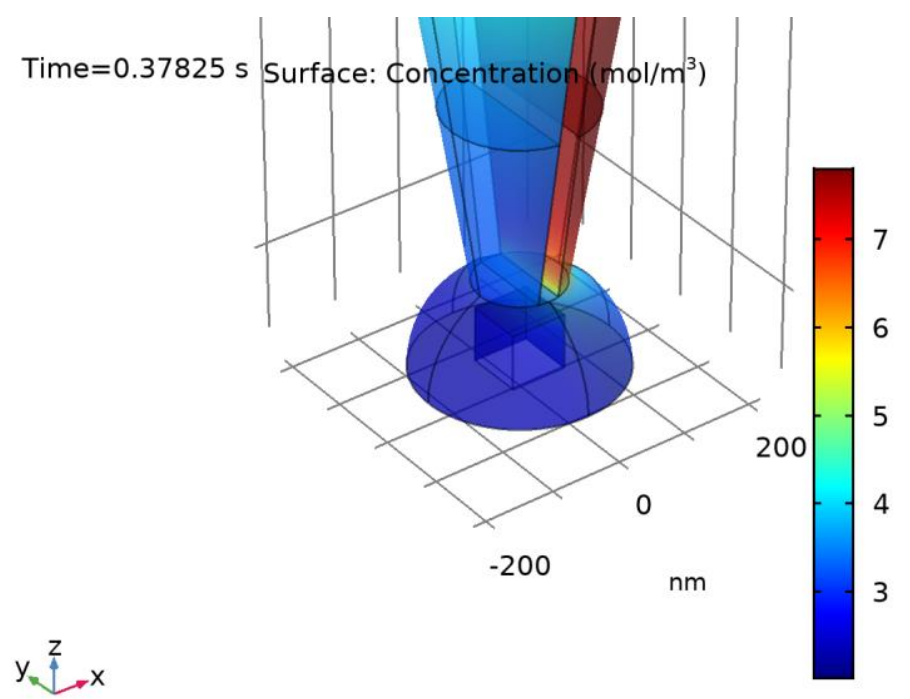

Surface: Concentration $\left(\mathrm{mol} / \mathrm{m}^{3}\right)$

Electric Potential (es)

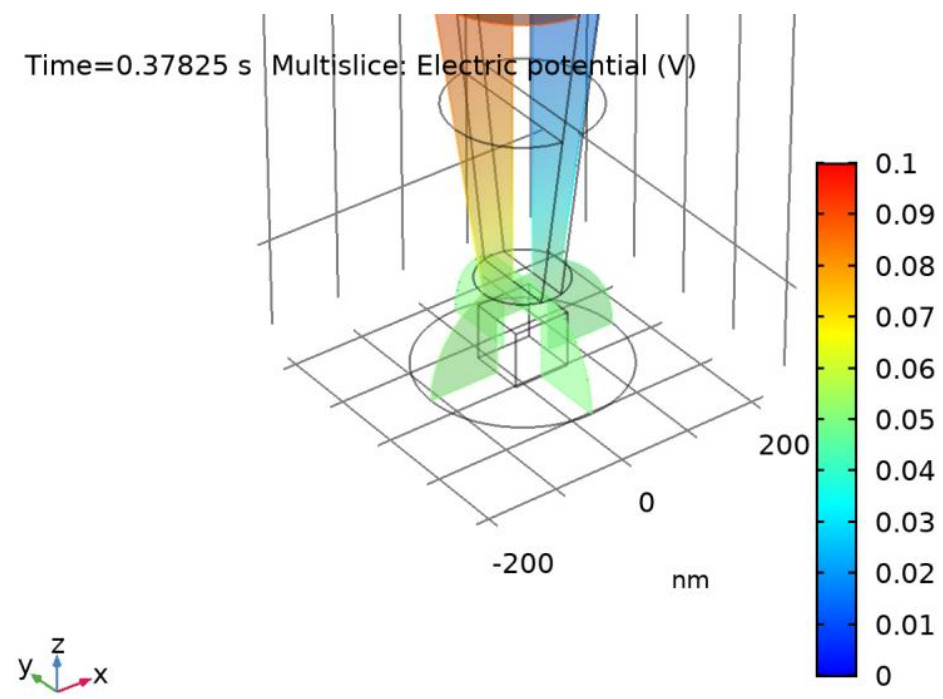

Multislice: Electric potential (V) 


\section{Supplementary Reference}

1. Nikoobakht, B.; El-Sayed, M. A. Preparation and growth mechanism of gold nanorods (NRs) using seed-mediated growth method. Chem. Mater. 2003, 15, 1957-1962.

2. O’Brien, M. N.; Jones, M. R.; Brown, K. A.; Mirkin, C. A. Universal noble metal nanoparticle seeds realized through iterative reductive growth and oxidative dissolution reactions. J. Am. Chem. Soc. 2014, 136, 7603-7606.

3. Niu, W.; Zheng, S.; Wang, D.; Liu, X.; Li, H.; Han, S.; Chen, J.; Tang, Z.; Xu, G. Selective synthesis of single-crystalline rhombic dodecahedral, octahedral, and cubic gold nanocrystals. J. Am. Chem. Soc. 2009, 131, 697-703.

4. $\quad$ Clark, E. L.; Hahn, C.; Jaramillo, T. F.; Bell, A. T. Electrochemical $\mathrm{CO}_{2}$ reduction over compressively strained $\mathrm{CuAg}$ surface alloys with enhanced multi-carbon oxygenate selectivity. $J$. Am. Chem. Soc. 2017, 139, 15848-15857.

5. Bentley, C. L.; Perry, D.; Unwin, P. R. Stability and placement of Ag/AgCl quasireference counter electrodes in confined electrochemical cells. Anal. Chem. 2018, 90, 77007707.

6. $\quad$ Ebejer, N.; Güell, A. G.; Lai, S. C. S.; McKelvey, K.; Snowden, M. E.; Unwin, P. R. Scanning electrochemical cell microscopy: A versatile technique for nanoscale electrochemistry and functional imaging. Annu. Rev. Anal. Chem. 2013, 6, 329-351.

7. Ebejer, N.; Schnippering, M.; Colburn, A. W.; Edwards, M. A.; Unwin, P. R. Localized high resolution electrochemistry and multifunctional imaging: Scanning electrochemical cell microscopy. Anal. Chem. 2010, 82, 9141-9145.

8. Lohrengel, M. M.; Moehring, A.; Pilaski, M. Capillary-based droplet cells: limits and new aspects. Electrochim. Acta 2001, 47, 137-141.

9. Snowden, M. E.; Güell, A. G.; Lai, S. C. S.; McKelvey, K.; Ebejer, N.; O’Connell, M. A.; Colburn, A. W.; Unwin, P. R. Scanning electrochemical cell microscopy: Theory and experiment for quantitative high resolution spatially-resolved voltammetry and simultaneous ionconductance measurements. Anal. Chem. 2012, 84, 2483-2491.

10. Novak, P.; Li, C.; Shevchuk, A. I.; Stepanyan, R.; Caldwell, M.; Hughes, S.; Smart, T. G.; Gorelik, J.; Ostanin, V. P.; Lab, M. J.; Moss, G. W. J.; Frolenkov, G. I.; Klenerman, D.; Korchev, Y. E. Nanoscale live-cell imaging using hopping probe ion conductance microscopy. Nat. Methods 2009, 6, 279.

11. Ushiki, T.; Nakajima, M.; Choi, M.; Cho, S.-J.; Iwata, F. Scanning ion conductance microscopy for imaging biological samples in liquid: A comparative study with atomic force microscopy and scanning electron microscopy. Micron 2012, 43, 1390-1398.

12. Jung, G.-E.; Noh, H.; Shin, Y. K.; Kahng, S.-J.; Baik, K. Y.; Kim, H.-B.; Cho, N.-J.; Cho, S.-J. Closed-loop ARS mode for scanning ion conductance microscopy with improved speed and stability for live cell imaging applications. Nanoscale 2015, 7, 10989-10997.

13. Ustarroz, J.; Ornelas, I. M.; Zhang, G.; Perry, D.; Kang, M.; Bentley, C. L.; Walker, M.; Unwin, P. R. Mobility and poisoning of mass-selected platinum nanoclusters during the oxygen reduction reaction. ACS Catal. 2018, 8, 6775-6790.

14. Saha, P.; Hill, J. W.; Walmsley, J. D.; Hill, C. M. Probing electrocatalysis at individual Au nanorods via correlated optical and electrochemical measurements. Anal. Chem. 2018, 90, 12832-12839. 
15. Heil, S. R.; Holz, M.; Kastner, T. M.; Weingärtner, H. Self-diffusion of the perchlorate ion in aqueous electrolyte solutions measured by ${ }^{35} \mathrm{Cl} \mathrm{NMR}$ spin-echo experiments. J. Chem. Soc., Faraday Trans. 1995, 91, 1877-1880.

16. Hamelin, A.; Weaver, M. J. Dependence of the kinetics of proton reduction at gold electrodes on the surface cyrstallographic orientation. J. Electroanal. Chem. 1987, 223, 171-184. 17. White, H. S.; Bund, A. Ion current rectification at nanopores in glass membranes. Langmuir 2008, 24, 2212-2218.

18. Angell, D. H.; Dickinson, T. The kinetics of the ferrous/ferric and ferro/ferricyanide reactions at platinum and gold electrodes: Part I. Kinetics at bare-metal surfaces. J. Electroanal. Chem. 1972, 35, 55-72.

19. Murray, R. C.; Rock, P. A. The determination of the ferrocyanide-ferricyanide standard electrode potential at $25^{\circ} \mathrm{C}$ in cells without liquid junction using cation-sensitive glass electrodes. Electrochim. Acta 1968, 13, 969-975. 